\title{
Integrative Analyses of Whole-Transcriptome \\ Sequencing Reveals CeRNA Regulatory Network of mRNAs, IncRNAs, miRNAs and circRNAs in Pulmonary Hypertension Treated with FGF21
}

\section{Xiuchun Li}

Wenzhou Medical University First Affiliated Hospital: The First Affiliated Hospital of Wenzhou Medical University

\section{Haijian Cai}

Wenzhou Medical University First Affiliated Hospital: The First Affiliated Hospital of Wenzhou Medical University

\section{Chi Zhang}

Wenzhou Medical College - Chashan Campus: Wenzhou Medical University

\section{Yaxin Zhang}

Wenzhou Medical College First Affiliated Hospital: The First Affiliated Hospital of Wenzhou Medical University

\section{Xinghong Wang}

Wenzhou Medical College First Affiliated Hospital: The First Affiliated Hospital of Wenzhou Medical University

\section{Lanlan Song}

Wenzhou Medical College First Affiliated Hospital: The First Affiliated Hospital of Wenzhou Medical University

Jianhao Zhang

Wenzhou Medical College - Chashan Campus: Wenzhou Medical University Jin Lin

Wenzhou Medical College - Chashan Campus: Wenzhou Medical University

\section{Shuolan Tong}

Wenzhou Medical College - Chashan Campus: Wenzhou Medical University

\section{Liangxing Wang}

Wenzhou Medical College First Affiliated Hospital: The First Affiliated Hospital of Wenzhou Medical University

\section{Xiaoying Huang ( $\nabla$ drhuangxy@163.com )}

Wenzhou Medical College First Affiliated Hospital: The First Affiliated Hospital of Wenzhou Medical University 


\section{Research Article}

Keywords: pulmonary hypertension, noncoding RNA, competing endogenous RNA, RNA sequencing, bioinformatics analysis

Posted Date: December 30th, 2021

DOI: https://doi.org/10.21203/rs.3.rs-1068049/v1

License: (c) (i) This work is licensed under a Creative Commons Attribution 4.0 International License. Read Full License 


\section{Abstract}

Noncoding RNAs have been shown to play important roles in hypoxic pulmonary hypertension (HPH). Our preliminary data showed that HPH is attenuated by fibroblast growth factor 21 (FGF21) administration. Therefore, we further investigated the whole transcriptome RNA expression patterns and interactions in a mice HPH model treated with FGF21. By whole-transcriptome sequencing, differentially expressed mRNA, miRNA, IncRNA, and circRNA were successfully identified in normoxia $(\mathrm{Nx})$ vs. hypoxia $(\mathrm{Hx})$ and $\mathrm{Hx} v s$. hypoxia + FGF21 (Hx + F21). Through intersection and predictive analysis, differentially co-expressed mRNA, miRNA, IncRNA, and circRNA were selected, followed by functional enrichment analysis. MAPK signaling pathway and epigenetic modification were enriched and may play fundamental roles in the therapeutic effects of FGF21. A ceRNA regulatory network was constructed with miR-7a-5p, miR-449c-5p, miR-676-3p and miR-674-3p as the core. Then the quantitative real time-PCR validation results were consistent with the results of whole-transcriptome sequencing. This study may provide potential biomarkers, pathway and ceRNA regulatory network in HPH treated with FGF21.

\section{Introduction}

As a subtype of pulmonary hypertension ( $\mathrm{PH}$ ) (group 3), hypoxic pulmonary hypertension (HPH) is an insidious and fatal disease with a poor long-term prognosis [1 2]. The reason for cure difficulty in HPH patients is the lack of accurate and noninvasive biomarkers for early detection, and no effective medication with limited side effects [3]. Therefore, it is highly urgent to explore potential biomarkers and novel therapeutic targets for HPH.

Recently, high-throughput next-generation sequencing (NGS) technologies have enabled our research to be no longer limited to messenger RNA (mRNA) expression profiles, but from mRNA to non-coding RNA, such as microRNA (miRNA), long non-coding RNA (IncRNA), and circular RNA (circRNA) [4 5]. With the deepening of research, non-coding RNAs have been found to play important roles in epigenetic regulation, transcriptional regulation, and posttranscriptional regulation, and participate in a variety of biological processes and diseases [6 7]. Competitive endogenous RNA (ceRNA) hypothesis mentioned by Salmena et al. states that mRNA, IncRNA, circRNA could act as miRNA sponge to modulate the stability or translational activity of target genes [8]. At present, the regulatory function of ceRNA has been widely accepted, and ceRNA pattern in HPH development has also been widely reported. As an example, Jiang Y et al. reported that circRNA Calm4 regulated hypoxia-induced pulmonary arterial smooth muscle cells (PASMCs) pyroptosis by ceRNA mechanism of sponging miR-124 [9]. However, few studies have delineated the transcriptomic landscape of HPH utilizing whole-transcriptome sequencing strategies.

Fibroblast growth factor 21 (FGF21) has been found to exert remarkable cardiovascular benefits in recent years. Cong Liu et al. found that pharmacological treatment with FGF21 could reduce atherosclerosis by the improvement of plasma cholesterol metabolism [10]. Xuebo Pan et al. uncovered that replenishment of FGF21 alleviated angiotensin II-Induced hypertension and vascular dysfunction by angiotensinconverting enzyme 2 (ACE2) in mice [11]. Our preliminary data indicated that exogenous administration 
of FGF21 relieved HPH and right ventricular hypertrophy [12]. However, the underlying mechanism in PH treated with FGF21 is thought to be complex, and systematic elucidation of the mechanisms and efficacy of FGF21 in HPH is therefore necessary for wider acceptance and clinical application.

In this study, we focused on the perturbation of hypoxia intervention and FGF21 administration on the non-coding RNA panorama in HPH. We first detected the expression of FGF21 in human and experimental $\mathrm{HPH}$, and selected the appropriate dose of FGF21 treatment group mice $(\mathrm{Hx}+\mathrm{F} 21)$, together with the normoxia ( $\mathrm{Nx}$ ) and hypoxia mice $(\mathrm{Hx})$, performed whole-transcriptome sequencing. Subsequently, differentially expressed mRNA, miRNA, IncRNA, and circRNA was screened between Nx and Hx groups, as well as $\mathrm{Hx}$ and $\mathrm{Hx}+\mathrm{F} 21$ groups, followed by functional enrichment analysis. Through intersection and predictive analysis, we finally anchored the differentially co-expressed mRNA, miRNA, IncRNA, and circRNA regulated by hypoxia and FGF21, constructed a ceRNA regulatory network, and performed qRTPCR verification. This research may provide potential biomarkers for the diagnosis and treatment of HPH, and provide a fundamental for clinical application of FGF21 in HPH.

\section{Material And Methods}

\section{Human clinical blood samples}

9 patients with high-altitude $\mathrm{PH}(\mathrm{HAPH})$ were enrolled between April 2019 and June 2019 at the First Affiliated Hospital of Wenzhou Medical University, and Qinghai Golmujianqiao Hospital. And 21 agematched healthy controls (HCs) were recruited. Blood samples were obtained from HAPH, or HCs in a quiet state. The blood was drawn into blood collection tubes, gently mixed and allowed to stand for $1 \mathrm{~h}$ at room temperature (RT). Then the tubes were centrifuged at $4^{\circ} \mathrm{C}$ within $2 \mathrm{~h}$ to separate the serum. The serum was immediately stored at $-80^{\circ} \mathrm{C}$ until use to avoid degradation. All procedures performed in studies involving human participants were approved by the Ethics Committee of the First Affiliated Hospital of Wenzhou Medical University.

\section{HPH models and drug applications}

Male C57BL/ 6 mice were purchased from the Animal Center of the Chinese Academy of Sciences (Shanghai, China). All animals started modeling after a week of rest. The construction of HPH mouse model was described previously [13]. Briefly, mice were randomly divided into five groups, each with 6 mice: normoxia ( $\mathrm{Nx}$, saline-treated), hypoxia ( $\mathrm{Hx}$, saline-treated), hypoxia plus FGF21 ( $\mathrm{Hx}+\mathrm{F} 21, \mathrm{~F} 21,50$, $100,200 \mu \mathrm{g} / \mathrm{kg} / \mathrm{day}$ ). Mice in the hypoxic group were kept in a normobaric hypoxic animal chamber (9$11 \%$ oxygen concentration) for 3 weeks. The normoxia group was exposed to indoor air. All groups were given adequate water and food. All animal work was approved by the Animal Ethics Committee of Wenzhou Medical University.

FGF21 was obtained from Prospec (CYT-281, Prospec, Rehovot, Israel). Briefly, FGF21 (1 mg) was dissolved in $10 \mathrm{ml} 0 \cdot 1 \%$ bovine serum albumin (BSA) solution. Then the medicine was injected intraperitoneally into mice at the above prescription dose per day before entering the hypoxia chamber. 
After 3 weeks, all mice were anesthetized and subjected to invasive hemodynamic measurements; then, the mouse blood, and lungs were collected for further study.

\section{Biochemical parameters}

Mouse serum FGF21, and human serum FGF21 levels were detected with an FGF21 Quantikine Elisa kit (R\&D Systems, Minneapolis, MN, USA), and a Human FGF21 immunoassay kit (ImmunoDiagnostics, Inc.), respectively. Blood glucose was measured using ACCU-CHEK active blood glucose meter.

\section{Measurements of invasive hemodynamic and RV hypertrophy}

Invasive hemodynamics measurements were measured as described [13]. We measured the mean right ventricular pressure (mRVP), and the mean carotid arterial pressure (mCAP) to evaluate the effectiveness of the model using PowerLab 8/35 multichannel biological signal recording system (AD Instruments, AUS). And the right ventricular wall, left ventricular wall and ventricular septum were separated to assess the hypertrophy of the right ventricle, and respond with two indicators: the ratio of right ventricle weight to body weight (RV/BW), and the ratio of right ventricle weight to left ventricle plus interventricular septum weight (RV/LV+S).

\section{Tissue staining}

4- $\mu$ m-thick paraffin-embedded lung tissue blocks were sectioned for staining experiment. HematoxylinEosin (HE) staining and Masson staining was both performed according to routine protocols. In bright field, we use a microscope (Nikon, Tokyo, Japan) to select typical pulmonary arterioles (outer diameter 25$100 \mathrm{~mm}$ ). The ratios of the wall thickness to the total thickness (WT/TT) and the ratios of the pulmonary artery wall area to the total area (WA/TA) were calculated to reflect pulmonary arterial remodeling. The ratio of the collagen area to the total area of the recording area was calculated to reflect the degree of collagen deposition. Immunohistochemical detection were used to determine FGF21 expression. Lung tissue sections were treated with citrate antigen repair buffer $(\mathrm{pH} 6.0)$ for antigen repair, then incubated with $0.3 \%$ hydrogen peroxide, and $5 \%$ BSA for blocking. Then, lung tissues were incubated with antiFGF21 antibody (diluted 1:200, ab171941, Abcam) overnight at $4^{\circ} \mathrm{C}$ and incubated for $1 \mathrm{~h}$ with horseradish peroxidase-conjugated goat anti-rabbit IgG (Zhong Shan Jin Qiao) next day. Subsequently, the lung sections were observed under Nikon microscope and brown-yellow particles were regarded as positive signals of the immunohistochemical staining.

\section{Western blot detection}

A Pierce BCA protein assay kit (Thermo Fisher Scientific Inc., Rockford, IL, USA) was used to quantify the total protein of mouse lung tissue homogenate or the total protein of rat pulmonary artery smooth muscle cells. After heat denaturation, it was prepared into 60-100 ug/ul protein standard and stored at $4^{\circ} \mathrm{C}$. The protein was fully separated by $10 \%$ SDS-polyacrylamide gel electrophoresis (SDS-PAGE), and transferred to a 0.22 um pore PVDF membrane, and then incubated in $5 \%$ skim milk at $37^{\circ} \mathrm{C}$ for $90 \mathrm{~min}$. After washing and cutting, the PVDG membrane was incubated in the primary antibody overnight at $4^{\circ} \mathrm{C}$. the 
secondary antibody of rabbit or mouse with goat antiserum labeled with horseradish peroxidase (1:

10000 , biosharp) was used to incubate at $37^{\circ} \mathrm{C}$ for 60 minutes, and then using ECL to exposure by (biorad exposure machine). The primary antibodies used were as follows: goat anti-rabbit IgG H\&L (1:10000, ab205718, Abcam), rabbit anti-FGF21 (1:1000, ab171941, Abcam), rabbit anti-PCNA (1:1000, \#13110, CST), rabbit anti- $\beta$-ACTIN (1:1000, \#4970, CST). Experiments were repeated at least 3 times and $\beta$-ACTIN was used as an internal control.

\section{RNA isolation and quantitative RT (qRT)-PCR.}

For mRNA, IncRNA and circRNAs, total RNA was extracted from the lung tissue or rPASMCs using Trizol (Invitrogen), and chloroform. For miRNAs, total RNA was extracted from the lung tissue using SanPrep Column microRNA Extraction Kit. For mRNA, IncRNA and circRNAs, relative cDNA was synthesized from 1 to $3 \mathrm{mg}$ of RNA using the iScript cDNA Synthesis Kit (Bio-Rad, USA). For miRNAs, relative cDNA was synthesized using miRNA First Strand cDNA Synthesis (Stem-loop Method) (Sangon Biotech, Shanghai, China). The mRNAs, IncRNAs and circRNAs expression were measured using qRT-PCR (CFX96 Real-Time System, Bio-Rad, USA). And the PCR for miRNAs was using microRNAs qPCR kit (Sangon Biotech, Shanghai, China). $\beta$-actin or U6 served as endogenous controls. The relative fold change was calculated using log2 FC. The primer sequences were shown in Table 1.

Table 1. Primers used for qRT-PCR in this study 


\begin{tabular}{|c|c|}
\hline Gene / Serial number & Primer sequence \\
\hline \multirow[t]{2}{*}{ FGF21 } & F: ATCAGGGAGGATGGAACAGTG \\
\hline & R: TTGGCAAAGAAACCTAGAGGC \\
\hline \multirow[t]{4}{*}{ mmu-miR-7a-5p } & F: GGGCTGGAAGACTAGTGATTT \\
\hline & RT:GTCGTATCCAGTGCAGGGTCCGAGGT \\
\hline & ATTCGCACTGGATACGACACAACA \\
\hline & R: CAGTGCAGGGTCCGAGGTAT \\
\hline \multirow[t]{4}{*}{ mmu-miR-449c-5p } & F: GGGCAGGCAGTGCATTGCT \\
\hline & RT:GTCGTATCCAGTGCAGGGTCCGAGGT \\
\hline & ATTCGCACTGGATACGACCCAGCT \\
\hline & R: CAGTGCAGGGTCCGAGGTAT \\
\hline \multirow[t]{4}{*}{ mmu-miR-674-3p } & F: GGGCCACAGCTCCCATCTCA \\
\hline & RT:GTCGTATCCAGTGCAGGGTCCGAGGT \\
\hline & ATTCGCACTGGATACGACTTGTTC \\
\hline & R: CAGTGCAGGGTCCGAGGTAT \\
\hline \multirow[t]{4}{*}{ mmu-miR-676-3p } & F: GGGCCCGTCCTGAGGTTGT \\
\hline & RT: GTCGTATCCAGTGCAGGGTCCGAGGT \\
\hline & ATTCGCACTGGATACGACAGCTCA \\
\hline & R: CAGTGCAGGGTCCGAGGTAT \\
\hline \multirow[t]{2}{*}{ Circ_Rbm27-108 } & F: AAACACCGTCTTGGACATGC \\
\hline & R: TGATTGTTGTTCCCTTGTCG \\
\hline \multirow[t]{2}{*}{ Circ_Mctp1-91 } & F: GAAAGAGGTGGGATCATGGA \\
\hline & R: CGTAAACTTTGGGTCTGAAACTTC \\
\hline \multirow[t]{2}{*}{ Circ_Gm672-96 } & F: CCTCAACATGCTCCAGACG \\
\hline & R: CCCTGTTGTGTTTGGCACT \\
\hline \multirow[t]{2}{*}{ Circ_Gfra2-80 } & F: GAGGACAAAGAGAAGCCCAAT \\
\hline & R: GAGCGGAGGGTTTCGTCGG \\
\hline \multirow[t]{2}{*}{ XR_375486.1-82 } & F: AAAAGTTCACATCCCCCAAA \\
\hline & R: CCTAGTTTGGCTTTGGCTGT \\
\hline XR_380261.1-115 & F: TACAACCCAACAGGGGAAGA \\
\hline
\end{tabular}




\begin{tabular}{|c|c|}
\hline & R: TGCCTCGCTTGTATTTCACA \\
\hline \multirow[t]{2}{*}{ XR_878320-83 } & F: TCTGAATGATGGTGGGTGAA \\
\hline & R: GCAAATCTGGGGTGAGAAGA \\
\hline \multirow[t]{2}{*}{ XR_881742.2-116 } & F: TCCACCAGCTCGAGAATAGC \\
\hline & R: TGCTGGCTATAGGAGGCAGT \\
\hline \multirow[t]{2}{*}{ XR_879969-94 } & F: CCCCTCGGTTGCTTTAAATA \\
\hline & R: ATAATTTCAAGGCGGCCATC \\
\hline \multirow[t]{2}{*}{ XR_867257-81 } & F: AAGCAATACAGCCCCTTGTG \\
\hline & R: AATAGCGTCATCGCTTTGCT \\
\hline \multirow[t]{2}{*}{ Col11a1-83 } & F: GGTGGAAAACGAAACGGTGG \\
\hline & R: TCTGACCTCTCTAGCTTGGAAG \\
\hline \multirow[t]{2}{*}{ Kcnj3-110 } & F: GGGGACGATTACCAGGTAGTG \\
\hline & R: CGCTGCCGTTTCTTCTTGG \\
\hline \multirow[t]{2}{*}{ Kcnj5-151 } & F: AAAACCTTAGCGGCTTTGTATCT \\
\hline & R: AAGGCATTAACAATCGAGCCC \\
\hline \multirow[t]{2}{*}{ Ptges3l-91 } & F: GGAATGGTGATGGAGTGGAG \\
\hline & R: GAGCGACCAGAACGCTTATC \\
\hline \multirow[t]{2}{*}{ Knstrn-93 } & F: GACTCGGAAACATGGGAAGA \\
\hline & R: AGAGAGACAAGGGGACAGCA \\
\hline \multirow[t]{2}{*}{ Cbfa2t3-102 } & F: CGTTTCCTCACCACСCTACAG \\
\hline & R: CGTCAGAGTTGAGTTCACCAG \\
\hline \multirow[t]{2}{*}{ Gltscr1-179 } & F: TGGGAGATGCTTACTAGACGTG \\
\hline & R: GCTCAGGGTTTAGGTGGTTGG \\
\hline \multirow[t]{2}{*}{ Ncor2-113 } & F: AACACCACCCCCGTGACTA \\
\hline & R: CTGAGACCGTTCACTCCCA \\
\hline \multirow[t]{2}{*}{ Satb2-101 } & F: GCCGTGGGAGGTTTGATGATT \\
\hline & R: ACCAAGACGAACTCAGCGTG \\
\hline \multirow[t]{2}{*}{ Atp1b2-181 } & F: GGCAGGTGGTTGAGGAGTG \\
\hline & R: GGGGTATGGTCAGAGACGGT \\
\hline
\end{tabular}


The whole transcriptome sequencing service of lung tissue of $\mathrm{HPH}$ animal models (5 mice in each group) were provided by RiboBio Co., Ltd (Guangzhou, China). Total RNA was extracted and prepared from lung tissue with Trizol reagent (Thermo Fisher Scientific, USA), and its purity and concentration were determined by ND-1000 Nanodrop. All samples meeting the A260/A280>1.8 and A260/A230>2.0 standards were used for subsequent analysis. RNA integrity was evaluated on the Agilent 2200 TapeStation, and those with an average RNA integrity number (RIN) greater than 7.0 were used for sequencing. The NEBNext ${ }^{\circledR}$ Ultra ${ }^{\text {TM }}$ RNA library was prepared using Illumina kits. RNA sequencing was performed by Illumina HiSeq 3000 , using $2 \times 150 \mathrm{bp}$ pair-end sequencing. The quality control of the original sequencing data was checked and processed by FASTQC software and Trimmomatic Tools. After comparing the sequencing data with the mouse reference genome mm10 using HISAT2 software, the reads mapped to each gene were calculated by HTSEQ. The raw data of RNA-seq was deposited in the NCBI Sequence Read Archive (SRA) under accession numbers, SRR SRR17180890-SRR17180934.

\section{Differential expression analysis}

For the whole transcriptome RNA sequencing results of three different treatments of mice, the "edgeR" $\mathrm{R}$ package [14] (3.34.1) was used for pairwise differential expression analysis ( $\mathrm{Nx} v s$. $\mathrm{Hx} ; \mathrm{Hx} v s$. $\mathrm{Hx}+\mathrm{F} 21)$. All transcripts that meet the p.value $<0.05, \| \log 2 \mathrm{FCl}>1$ screening threshold are considered significant. Through the "ggplot" package, the volcano map visually displays dif-mRNAs, dif-miRNAs, dif-IncRNAs and dif-circRNAs, respectively. The "heatmap" R package was used to draw cluster heatmaps of the top 100 RNAs in $\mid \log 2 \mathrm{FCl}$ among the four transcripts in three different treatment groups.

\section{Enrichment analysis}

In this article, all gene enrichment analysis was performed by the "clusterprofiler" R package (v3.16.1). Gene set enrichment analysis (GSEA) is a powerful method to enrich gene expression profiles based on the ranking of differential expression results to gain in-depth understanding of biological mechanisms. We download "c2.cp.kegg.v7.4.symbols.gmt" and "h.all.v7.4.symbols.gmt" from MSigDB as Kyoto Encyclopedia of Genes and Genomes (KEGG) pathway and hallmark gene reference set. The log2 FC obtained by the differential expression analysis of $\mathrm{Nx}$ vs $\mathrm{Hx}$ and $\mathrm{Hx}$ vs $\mathrm{Hx}+\mathrm{F} 21$ was used as the ranking list for GSEA analysis. All results of GSEA were visualized by "enrichplot" $\mathrm{R}$ package.

dif-genes that were up-regulated in $\mathrm{Hx}$ group compared with $\mathrm{Nx}$ group, and down-regulated in $\mathrm{Hx}+\mathrm{F} 21$ group compared with $\mathrm{Hx}$ group were recognized as co-expressed dif-genes. Conversely, dif-genes with another co-regulatory relationship were also selected as co-expressing dif-genes. The co-expressed difgenes obtained above were used for KEGG and Gene Ontology (GO) enrichment analysis. Before analysis, org.Mm.eg.db(http://www.bioconductor.org/packages/release/data/annotation/html/org.Mm.eg.db.html, 3.14.0) was used to annotate and map dif-genes. The bubble charts drawn by the "ggplot2" R package were used to display the enrichment results of KEGG and GO, among which GO result displayed the top 10 enriched items in biological process (BP), cellular component (CC), and molecular function (MF). And p.value $<0.05$ was considered significant. 


\section{Prediction of co-expressions dif-miRNA regulation relationship}

Three credible online databases: Targetscan (http://www.targetscan.org/) [15], miRDB (http://mirdb.org/) [16], and miRWalk 3.0 (http://mirwalk.umm.uni-heidelberg.de/) [17], were used to predicted the miRNAmRNA pairs. The screening principle of co-expressed dif-miRNA was consistent with the above-mentioned principle of co-expressed dif-genes. Co-expressed dif-miRNA were performed to predict target mRNAs. After the target genes predicted by the three databases were combined, they were further integrated with co-expressed dif-genes to obtain the regulatory relationship of dif-miRNA-dif-genes.

We retrieved dif-IncRNA and dif-circRNA sequences from the NCBI (https://www.ncbi.nlm.nih.gov/) and Ensembl database (http://www.ensembl.org/), co-expressed dif-miRNA sequences were obtained from miRbase (https://mirbase.org/) [18]. The interactions of dif-circRNA-dif-miRNA and dif-IncRNA-dif-miRNA were predicted by miRanda (http://www.microrna.org/microrna/home.do), and the results with a score higher than 140 and maximum binding free energy lower than $-10 \mathrm{kcal} / \mathrm{mol}$ were considered meaningful.

Finally, we focused on screening co-expressed dif-miRNAs which can simultaneously regulate difIncRNAs, dif-circRNAs, and dif-genes for subsequent construction of ceRNA networks. It is worth noting that $\| \log 2 \mathrm{FCl}>1$ was too harsh for the RNA-Seq results of circRNA, so we relaxed the standard for circRNA when screening dif-circRNA-dif-miRNA regulatory relationships. We only cared about p.value $<0.05$, and the threshold of $\mid \log 2 \mathrm{FCl}$ was not required [19]. The regulatory relationship will be experimentally verified in the follow-up qRT-PCR.

\section{Enrichment analysis of target genes regulated by co- expression dif-miRNAs}

In order to further explore the biological role of these co-expressed dif-miRNAs in the process of FGF21 treatment of $\mathrm{HPH}$, we performed KEGG and GO enrichment analysis on the target genes they regulate. As before, the "clusterprofiler" R package was responsible for enrichment analysis, and the "ggplot2" R package was responsible for visualization, p.value $<0.05$ was considered as significant enrichment.

\section{Construction of the dif-circRNA/dif-IncRNA-dif-miRNA-dif- genes network}

To investigate whole transcriptome ceRNA regulation in HPH, the dif-circRNA/dif-IncRNA-dif-miRNA-difgenes ceRNA network was built based on the miRNA sponge hypothesis [20], which proposed that IncRNAs and circRNAs could act as miRNA sponges to interact with miRNAs and regulate downstream mRNAs expression. Thus, on the basis of previous selection, we further screen dif-IncRNAs/dif-circRNAs and dif-genes that have the same variation trend, their corresponding miRNAs which have the opposite trend were chosen simultaneously. RNAs that fulfilled the above condition were combined to construct a dif-circRNA/dif-IncRNA-dif-miRNA-dif-genes ceRNA network in Nx vs. Hx and $\mathrm{Hx} v s$. Hx + F21, respectively. In order to further reflect the therapeutic effect of the ceRNA network regulated by FGF21 on PH, we 
screened the ceRNA network relationships that exist simultaneously in $\mathrm{Nx} v s$. $\mathrm{Hx}$ and $\mathrm{Hx} v s$. $\mathrm{Hx}+\mathrm{F} 21$. And these networks were integrated and constructed to obtain the final co-expressed ceRNA network of the whole transcriptome. Cytoscape v3.7.1 [21] was applied to display the network results.

\section{Statistical analysis}

All Statistical analyses were performed with GraphPad Prism 9.0. All data were expressed as mean \pm standard error mean (SEM). Comparisons between two groups were analyzed by unpaired two-tailed Student's t-test. Multiple comparisons were analyzed by ANOVA followed by Dunnett post-hoc test. P.value of $<0.05$ were considered statistically significant.

\section{Result}

\section{Expression of FGF21 is decreased in human and experimental PH.}

In this study, hypoxia-induced rPASMCs, and mouse PH models were established to determine the pathophysiological role of FGF21 in PH. Significantly lower FGF21 mRNA, and protein levels in rPASMC suspensions, and lung homogenates were detected after exposure to hypoxia (Figure. 1A-D). FGF21 was diffusely distributed, mainly in the vascular cell periphery (Figure. 1E). Circulating FGF21 levels in HPH mice, and HAPH patients were further examined. Consistent with the decline of serum FGF21 levels in HPH mice, circulating FGF21 levels were notably decreased in subjects with HAPH (Figure. 1F-G). These data support findings from our previous research [13 22] and demonstrate that FGF21 is downregulated in HAPH patients and plays a role in $\mathrm{PH}$ disease.

\section{PH amelioration in FGF21 treated mice.}

To further investigate the role of FGF21 in HPH, the optimal dose of human recombinant FGF21 was determined by evaluating its effect in HPH mice administered different concentrations (50, 100, and 200 $\mu \mathrm{g} / \mathrm{kg}$, intraperitoneally). In hypoxia-exposed mice subjected to daily administration of FGF21 at a dose of $200 \mu \mathrm{g} / \mathrm{kg}, \mathrm{mRVP}$ decreased significantly whereas the mCAP was unaffected, compared with that in hypoxia-exposed vehicle-treated mice (Figure. 2A-D). As anticipated, the increases in RV/(S+LV), and RV/WT under hypoxic conditions were markedly reduced by treatment with FGF21 (200 $\mu \mathrm{g} / \mathrm{kg})$ (Figure. $2 \mathrm{E}-\mathrm{F})$. Therefore, the optimal dose was $200 \mu \mathrm{g} / \mathrm{kg}$. FGF21 at a dose of $200 \mu \mathrm{g} / \mathrm{kg}$ did not influence the blood glucose in HPH mice (Figure. S1A). The western blot analysis, and immunohistochemical analysis (Figure. S1B-C) revealed that FGF21 expression was successfully increased after treatment with FGF21 $(200 \mu \mathrm{g} / \mathrm{kg})$. Furthermore, treatment with FGF21 also significantly attenuated hypoxia-induced pulmonary artery muscularization, as indicated by the ratios of WA/TA (\%), and WT/TT (\%) (Figure. 2G-I). The expression of PCNA in lung of FGF21-treated mice exposed to hypoxia was markedly decreased compared to that in the hypoxia group without FGF21 treatment (Figure. 2J-K). Additionally, the negative effects of excessive depositions of collagen around the pulmonary arteries were reversed by replenishment of FGF21 in HPH mice (Figure. 2L-M). Collectively, these results suggest that treatment with FGF21 reverses $\mathrm{PH}$. 
Differential expression analyses of mRNAs/miRNAs/IncRNAs/circRNAs in Nx vs. Hx and Hx vs. Hx + F21.

In light of the good performance of FGF21 in attenuating PH, we perform the whole-transcriptome sequencing on mice lung tissues from $\mathrm{Nx}, \mathrm{Hx}$ and $\mathrm{Hx}+\mathrm{F} 21$ groups to uncover the underlying mechanisms. According to the screening criteria, a total of 1396 (1126 up and 270 down) differentially expressed mRNAs (dif-mRNAs), 28 (18 up and 10 down) differentially expressed miRNAs (dif-miRNAs), 1263 (891 up and 372 down) differentially expressed IncRNAs (dif-IncRNAs), and 48 (28 up and 20 down) differentially expressed circRNAs (dif-circRNAs) were detected between Nx and Hx (Figure S2, DATA S1, S2, S3, S4). In this context, up indicates RNAs upregulated in $\mathrm{Hx}$, compared with $\mathrm{Nx}$. Comparing $\mathrm{Hx}$ with $\mathrm{Hx}+\mathrm{F} 21$, we found 1348 (198 up and 1150 down) differentially expressed mRNAs (dif-mRNAs), 20 (11 up and 9 down) differentially expressed miRNAs (dif-miRNAs), 1027 (269 up and 758 down) differentially expressed IncRNAs (dif-IncRNAs), and 13 (6 up and 7 down) differentially expressed circRNAs (difcircRNAs). In this context, up indicates RNAs upregulated in $\mathrm{Hx}+\mathrm{F} 21$, compared with $\mathrm{Hx}$ (Figure S3, DATA S5, S6, S7, S8). We then performed the hierarchical clustering heatmaps on the top 100 dif-mRNAs, difmiRNAs, dif-IncRNAs, and dif-circRNAs from all three groups as shown in Figure 3 . The results showed that $\mathrm{Hx}$ samples can be significantly separated from $\mathrm{Nx}$ and $\mathrm{Hx}+\mathrm{F} 21$ samples, indicating the analysis results were reliable.

GSEA of differential expressed genes in Nx vs. $\mathrm{Hx}$ and $\mathrm{Hx}$ vs. $\mathrm{Hx}+\mathrm{F} 21$.

GSEA was performed using the Hallmark and KEGG gene sets to explore the biological functions of dysregulated mRNAs. Comparing $\mathrm{Hx}$ with $\mathrm{Nx}$, GSEA showed that a total of five KEGG gene sets (Figure 4A) and eight hallmark gene sets (Figure 4C) were significantly enriched. Comparing $\mathrm{Hx}+\mathrm{F} 21$ with $\mathrm{Hx}$, GSEA showed that a total of three KEGG gene sets (Figure 4B) and four hallmark gene sets (Figure 4D) were significantly enriched. Overall, KEGG gene sets cell cycle and pyrimidine metabolism, Hallmark gene sets $\mathrm{E} 2 \mathrm{~F}$ targets, G2M checkpoint and MYC targets were downregulated in $\mathrm{Hx}$, compared with Nx while these gene sets were all upregulated in $\mathrm{Hx}+\mathrm{F} 21$, compared with $\mathrm{Hx}$ (Figure 4A-B). Moreover, KEGG gene sets endocytosis, and Hallmark gene sets estrogen response early were upregulated in $\mathrm{Hx}$, compared with $\mathrm{Nx}$ while these gene sets were all downregulated in $\mathrm{Hx}+\mathrm{F} 21$, compared with $\mathrm{Hx}$ (Figure 4C-D).

\section{Screening of differentially co-expressed molecules and functional enrichment analysis.}

To investigate the common and unique differential RNAs among all samples, we constructed venn diagrams. The results showed that 47 dif-genes, 5 dif-miRNAs, 113 dif-IncRNAs, and 11 dif-circRNAs were downregulated in $\mathrm{Hx}$, compared with $\mathrm{Nx}$; these co-expressed RNAs were upregulated in $\mathrm{Hx}+\mathrm{F} 21$, compared with Hx (Figure 5A-D, Data S9, S10, S11, S12). Moreover, 321 dif-genes, 6 dif-miRNAs, 520 difIncRNAs, and 7 dif-circRNAs were upregulated in $\mathrm{Hx}$, compared with $\mathrm{Nx}$; these co-expressed RNAs were all downregulated in $\mathrm{Hx}+\mathrm{F} 21$, compared with $\mathrm{Hx}$ (Figure 5A-D, Data S9, S10, S11, S12). Then we perform KEGG and GO analysis on the co-expressed dif-genes. KEGG pathway analysis showed that KEGG pathway such as cardiac muscle contraction, adrenergic signaling in cardiomyocytes and lysine degradation may be in volved in the effect of FGF21 in alleviating PH (Figure 5E). Moreover, GO terms such as covalent chromatin modification, histone modification and peptidyl-lysine modification of BP; 
nuclear chromatin, transcription regulator complex and transmembrane transporter complex of CC; transcription coregulator activity, RNA polymerase II-specific and DNA-binding transcription activator activity of MF were potentially associated with therapeutic effect of FGF21 (Figure 5F), which indicating that FGF21 may have a potential role in modulating transcriptional activity and the epigenetic landscape.

\section{MAPK signaling pathway may play an important role in HPH treated with FGF21.}

The target genes for 11 co-expression dif-miRNAs ( 5 down and 6 up, in $\mathrm{Hx}$, compared with $\mathrm{Nx}$ or $\mathrm{Hx}+$ F21) came from the intersection of three database prediction results and co-expressed dif-genes among three groups as shown in Figure 6A-B and Data S13. Comparing $\mathrm{Hx}$ with $\mathrm{Nx}$, there were 243 target genes. KEGG pathway analysis on these genes showed that MAPK signaling pathway was the most significantly enriched pathway (Figure 6C). GO analysis revealed that regulation of ion transmembrane transport, positive regulation of cell projection organization and lung epithelial cell differentiation were enriched (Figure 6D). Comparing Hx + F21 with Hx, there were 215 target genes. MAPK signaling pathway was also the most significant enriched pathway from KEGG analysis on these target genes (Figure 6E). As for GO BP terms, histone modification, covalent chromatin modification and regulation of transporter activity were significantly enriched (Figure 6F). These observations support the enrichment analysis results of the co-expressed dif-genes as shown in Figure 5E-F.

\section{Construction of the ceRNA network in $\mathrm{Nx}$ vs. $\mathrm{Hx}$ and $\mathrm{Hx}$ vs. $\mathrm{Hx}+\mathrm{F} 21$.}

The dif-miRNA-dif-gene regulatory relationship was obtained according to the results of Figure 6A-B and Data S13. The dif-miRNA-dif-IncRNA (Data S14) and dif-miRNA-dif-circRNA (Data S15) regulatory relationships were predicted using the miRanda database. Using dif-miRNA as a bridge, we construct circRNA- or IncRNA-miRNA-gene network based on dif-miRNA-dif-gene, dif-miRNA-dif-IncRNA, and difmiRNA-dif-circRNA regulatory relationships. Comparing $\mathrm{Hx}$ with $\mathrm{Nx}$, the downregulated-dif-miRNAassociated ceRNA network comprised 5 dif-miRNAs, 172 dif-genes, 320 dif-IncRNAs, and 18 dif-circRNAs (Figure. 7A, Data S16); the upregulated-dif-miRNA-associated ceRNA network comprised 3 dif-miRNAs, 50 dif-genes, 99 dif-IncRNAs, and 10 dif-circRNAs (Figure. 7B, Data S16). Comparing $\mathrm{Hx}+\mathrm{F} 21$ with $\mathrm{Hx}$, the downregulated-dif-miRNA-associated ceRNA network comprised 3 dif-miRNAs, 42 dif-genes, 42 difIncRNAs, and 4 dif-circRNAs (Figure. 8A, Data S17); the upregulated-dif-miRNA-associated ceRNA network comprised 5 dif-miRNAs, 169 dif-genes, 209 dif-IncRNAs, and 8 dif-circRNAs (Figure. 8B, Data S17).

\section{Construction of the co-expressed ceRNA network.}

To explore the co-expressed ceRNA network regulated by FGF21, we next took the intersection of the upregulated-dif-miRNA-associated ceRNA network in $\mathrm{Hx} v$ s. $\mathrm{Nx}$, and the downregulated-dif-miRNAassociated ceRNA network in $\mathrm{Hx}+\mathrm{F} 21$ vs. Hx. There were 2 dif-miRNAs, 16 dif-genes, 16 dif-IncRNAs, and 2 dif-circRNAs to be found. Also, the downregulated-dif-miRNA-associated ceRNA network in $\mathrm{Hx} v s$. Nx, and the upregulated-dif-miRNA-associated ceRNA network in $\mathrm{Hx}+\mathrm{F} 21$ vs. $\mathrm{Hx}$ were taken to intersect. There were 2 dif-miRNAs, 79 dif-genes, 113 dif-IncRNAs, and 2 dif-circRNAs to be found. Cytoscape was applied to build the co-expressed ceRNA network (Figure 9, Data S18). These integrated core ceRNA 
network revealed potential co-expression relationships of genes, IncRNAs, and circRNAs regulated by these miRNAs in PH treated with FGF21.

\section{QRT-PCR validation of differentially expressed RNAs in the core ceRNA network.}

Based on the core ceRNA network among the three groups, we chose the top 5 dif-RNAs for qRT-PCR verification. As shown in Figure. 10, it was confirmed that the expression of four miRNAs (two upregulated miRNA, miR-674 and miR-676; two downregulated miRNA, miR-7a and miR-449; in Hx compared with $\mathrm{Nx}$ and $\mathrm{Hx}+\mathrm{F} 21$ ), five IncRNAs (one upregulated IncRNA, XR_878320.1; three downregulated IncRNA, XR_375486.1, XR_867257.2, and XR_380261.1; in Hx compared with Nx and Hx + F21), four circRNAs (two upregulated circRNAs, circ_Rbm27 and circ_mctp1; two downregulated circRNAs, circ_Gm672 and circ_Gfra2; in Hx compared with Nx and Hx + F21), and six mRNAs (one upregulated mRNAs, Atp1b2; five downregulated mRNAs, Col11a1, Kcnj3, Kcnj5, Knstrn, and Ptges3l), these results were consistent with the RNA-Seq data, which indicated that our study was convincing.

\section{Discussion}

FGF21 is a well-established vascular protective factor [ 1023 24], and changes in circulating FGF21 levels are closely related to many diseases, such as subclinical atherosclerosis [25], alcoholic cardiomyopathy [26], and remyelination in the central nervous system [27]. Our research showed that the reduction of FGF21 level in the serum of HAPH patients, as well as in the serum and lung tissue of mice HPH model, which suggesting a strong correlation between the levels of circulating and lung FGF21 and HPH. Administration of FGF21 alleviated the progression of HPH in our research, which is consistent with the vascular benefit of FGF21 reported by most researchers, and also broadens the application of FGF21 in the pulmonary circulation.

However, the mechanism underlying the therapeutic effect of FGF21 is complicated. We assumed that epigenetics may play an important role in its anti-vascular remodeling properties. By using wholetranscriptome sequencing, we identified a total of 1396 dif-mRNAs, 28 dif-miRNAs, 1263 dif-IncRNAs, and 48 dif-circRNAs in $\mathrm{Hx}$ compared with $\mathrm{Nx}$, and a total of 1348 dif-mRNAs, 20 dif-miRNAs, 1027 difIncRNAs, and 13 dif-circRNAs in $\mathrm{Hx}+\mathrm{F} 21$ compared with $\mathrm{Hx}$. Enrichment analysis on the dif-mRNAs showed that cell cycle and G2M checkpoint might participate in the regulation of hypoxia, and the protective effect of FGF21 under hypoxic stimulation might be related to its mediation of cell endocytosis. Shuang-Lan Xu et al. [28] and Jie Liu et al. [29] identified the differentially expressed circRNA/miRNA/mRNA and IncRNA/miRNA/mRNA in rat HPH model compared with controls, respectively. Their target genes were enriched in immune and inflammation-related pathways. Quite different from the rat model, in this study, we focus on mice models. Compared with immune and inflammatory effects, cell cycle may be more related to the pathology of vascular remodeling of HPH [30 31]. In addition, we used whole-transcriptome sequencing to construct a dif-circRNA/dif-IncRNA-difmiRNA-dif-genes interaction ceRNA network landscape, which more comprehensively demonstrated the 
relationship between these transcripts in the ceRNA network of HPH mice, also provided potential biomarkers for predicting new therapeutic targets for $\mathrm{HPH}$.

In addition to the disturbance of hypoxia, we paid more attention to the influence of FGF21. Through the intersection of the three groups, we focused on identifying the differentially expressed RNA molecules and enriched signaling pathways of their target genes during the course of disease development and FGF21 treatment. The result of miRNA target gene function enrichment showed that the chromatin modification, histone modification and peptidyl-lysine modification was affected after FGF21

intervention, which verified our hypothesis that epigenetics might be involved in FGF21 regulation. In this regard, Sangwon Byun et al. [32] reported that FGF21 promoted Jumonji-D3 (JMJD3/KDM6B) histone demethylase, thereby activated hepatic autophagy and lipid degradation. Teresa Płatek et al. [33] showed that DNA methylation changes and miRNA pattern play an important role in FGF21 resistance in obesity. Moreover, our data showed that MAPK signaling was involved in the process of HPH treated by FGF21, and the relationship between FGF21 and MAPK signaling has been reported in many articles [34-36].

In the ceRNA network constructed in our study, miRNAs are the bridges as well as the core factor. For the two RNAs upregulated by hypoxia, miRNA-674 could be induced by HIF-1a [37] and was upregulated in chronic obstructive pulmonary disease rats [38], which were closely related to $\mathrm{HPH}$; miR-676 was suggested as a potential therapeutic target in heart failure [39], suggesting that miR-676 might affect ventricular remodeling. These all support our observations. For the other two RNAs downregulated by hypoxia, miR-449 suppressed cell proliferation and promoted apoptosis [40], suggesting that FGF21induced upregulation of miR-449 expression might promote the homeostasis of pulmonary vascular structure through similar regulation; the overexpression of miR-7-5p suppressed the growth and cancer metabolism of lung cancer [41], and HPH is characterized by enhanced proliferation of vascular smooth muscle cell that shares similar pathophysiological processes with cancer cell. Due to space limitations, we cannot discuss the RNA molecular in core ceRNA one by one. Subsequent qRT-PCR verification showed that our analysis was reliable. Future studies are warranted to ascertain further this association.

In conclusion, this study explored the potential molecular mechanism of HPH and the FGF21-treated HPH using whole-transcriptome sequencing. Epigenetic modification and MAPK signaling may play fundamental roles in the pathogenesis of $\mathrm{HPH}$, and may account for the therapeutic effect of FGF21. FGF21 may reduce hypoxia-induced pathological deterioration through a ceRNA network with miR-7a, miR-449, miR-676 and miR-674 as the core. These findings suggest potential new targets in HPH characterization and FGF21 therapy. However, this study is limited by the small sample size, and further in vitro and in vivo verification is required to delineate the exact mechanism details.

\section{Declarations}

\section{Data availability statement}

The raw data presented in this study will be made available by the authors, without undue reservation. Sequencing data used in this manuscript can be found in the NCBI database SRA (PRJNA785350) and 
the SRR accession numbers are SRR17180890 to SRR17180934.

\section{Author contributions}

X.H., L.W. and X.L. contributed to the design of the study. X.L. and H.C performed the experiments in vivo and in vitro. X.L., C.Z., Y.Z., X.W., L.S. contributed to the interpretation of the data and organize the pictures. X.L., C.Z., J.Z., J.L. and S.T. executed the bioinformatics analysis and prepared the tables and figures. X.L., H.C and C.Z. wrote the manuscript. X.H. and L.W. contributed to modified the original manuscript and provided funding for the study. All authors approved the final version of the manuscript.

\section{Funding}

This work was supported by Chinese National Natural Science Foundation $(82170061,81873411)$ and college student innovation and entrepreneurship training program of China (202010343033).

\section{Declaration of interests}

The authors have declared that no competing interest exists.

\section{Acknowledgement}

None.

\section{Declaration of interests}

The authors have declared that no competing interest exists.

\section{References}

1. Luo Y, Dong HY, Zhang B, et al. miR-29a-3p attenuates hypoxic pulmonary hypertension by inhibiting pulmonary adventitial fibroblast activation. Hypertension 2015;65(2):414-20 doi:

10.1161/hypertensionaha.114.04600.

2. Xing Y, Zheng X, Fu Y, et al. Long Noncoding RNA-Maternally Expressed Gene 3 Contributes to Hypoxic Pulmonary Hypertension. Mol. Ther. 2019;27(12):2166-81 doi:

10.1016/j.ymthe.2019.07.022.

3. Hu CJ, Poth JM, Zhang H, et al. Suppression of HIF2 signalling attenuates the initiation of hypoxiainduced pulmonary hypertension. Eur. Respir. J. 2019;54(6) doi: 10.1183/13993003.00378-2019.

4. Bernardo A, St Amand P, Le HQ, Su Z, Bai G. Multiplex restriction amplicon sequencing: a novel nextgeneration sequencing-based marker platform for high-throughput genotyping. Plant biotechnology journal 2020;18(1):254-65 doi: 10.1111/pbi.13192.

5. Luthra R, Patel KP, Routbort MJ, et al. A Targeted High-Throughput Next-Generation Sequencing Panel for Clinical Screening of Mutations, Gene Amplifications, and Fusions in Solid Tumors. J. Mol. Diagn. 2017;19(2):255-64 doi: 10.1016/j.jmoldx.2016.09.011. 
6. Toden S, Zumwalt TJ, Goel A. Non-coding RNAs and potential therapeutic targeting in cancer. Biochimica et biophysica acta. Reviews on cancer 2021;1875(1):188491 doi: 10.1016/j.bbcan.2020.188491.

7. DeOcesano-Pereira C, Machado RAC, Chudzinski-Tavassi AM, Sogayar MC. Emerging Roles and Potential Applications of Non-Coding RNAs in Glioblastoma. Int. J. Mol. Sci. 2020;21(7) doi: 10.3390/ijms21072611.

8. Vitiello M, Evangelista M, Zhang Y, Salmena L, Pandolfı PP, Poliseno L. PTENP1 is a ceRNA for PTEN: it's CRISPR clear. J. Hematol. Oncol. 2020;13(1):73 doi: 10.1186/s13045-020-00894-2.

9. Jiang Y, Liu H, Yu H, et al. Circular RNA Calm4 Regulates Hypoxia-Induced Pulmonary Arterial Smooth Muscle Cells Pyroptosis via the Circ-Calm4/miR-124-3p/PDCD6 Axis. Arterioscler. Thromb. Vasc. Biol. 2021;41(5):1675-93 doi: 10.1161/atvbaha.120.315525.

10. Liu C, Schönke M, Zhou E, et al. Pharmacological treatment with FGF21 strongly improves plasma cholesterol metabolism to reduce atherosclerosis. Cardiovasc. Res. 2021 doi: 10.1093/cvr/cvab076.

11. Pan X, Shao Y, Wu F, et al. FGF21 Prevents Angiotensin II-Induced Hypertension and Vascular Dysfunction by Activation of ACE2/Angiotensin-(1-7) Axis in Mice. Cell Metab. 2018;27(6):1323-37.e5 doi: 10.1016/j.cmet.2018.04.002.

12. Liu J, Cai G, Li M, et al. Fibroblast growth factor 21 attenuates hypoxia-induced pulmonary hypertension by upregulating PPARy expression and suppressing inflammatory cytokine levels. Biochem. Biophys. Res. Commun. 2018;504(2):478-84 doi: 10.1016/j.bbrc.2018.09.004.

13. Cai G, Liu J, Wang M, et al. Mutual promotion of FGF21 and PPARY attenuates hypoxia-induced pulmonary hypertension. Exp. Biol. Med. (Maywood) 2019;244(3):252-61 doi: $10.1177 / 1535370219828692$.

14. Robinson MD, McCarthy DJ, Smyth GK. edgeR: a Bioconductor package for differential expression analysis of digital gene expression data. Bioinformatics 2010;26(1):139-40 doi: 10.1093/bioinformatics/btp616.

15. Agarwal V, Bell GW, Nam JW, Bartel DP. Predicting effective microRNA target sites in mammalian mRNAs. eLife 2015;4 doi: 10.7554/eLife.05005.

16. Chen $Y$, Wang $X$. miRDB: an online database for prediction of functional microRNA targets. Nucleic Acids Res. 2020;48(D1):D127-d31 doi: 10.1093/nar/gkz757.

17. Sticht C, De La Torre C, Parveen A, Gretz N. miRWalk: An online resource for prediction of microRNA binding sites. PLoS One 2018;13(10):e0206239 doi: 10.1371/journal.pone.0206239.

18. Griffiths-Jones S, Grocock RJ, van Dongen S, Bateman A, Enright AJ. miRBase: microRNA sequences, targets and gene nomenclature. Nucleic Acids Res. 2006;34(Database issue):D140-4 doi: 10.1093/nar/gkj112.

19. Zhou M, Hu L, Zhang Z, Wu N, Sun J, Su J. Recurrence-Associated Long Non-coding RNA Signature for Determining the Risk of Recurrence in Patients with Colon Cancer. Molecular therapy. Nucleic acids 2018;12:518-29 doi: 10.1016/j.omtn.2018.06.007. 
20. Thomson DW, Dinger ME. Endogenous microRNA sponges: evidence and controversy. Nature reviews. Genetics 2016;17(5):272-83 doi: 10.1038/nrg.2016.20.

21. Shannon P, Markiel A, Ozier O, et al. Cytoscape: a software environment for integrated models of biomolecular interaction networks. Genome Res. 2003;13(11):2498-504 doi: 10.1101/gr.1239303.

22. Liu J, Cai G, Li M, et al. Fibroblast growth factor 21 attenuates hypoxia-induced pulmonary hypertension by upregulating PPARgamma expression and suppressing inflammatory cytokine levels. Biochem. Biophys. Res. Commun. 2018;504(2):478-84 doi: 10.1016/j.bbrc.2018.09.004.

23. Tomita Y, Fu Z, Wang Z, et al. Long-Acting FGF21 Inhibits Retinal Vascular Leakage in In Vivo and In Vitro Models. Int. J. Mol. Sci. 2020;21(4) doi: 10.3390/ijms21041188.

24. Wang HW, Jiang X, Zhang Y, et al. FGF21 Protects Against Hypoxia Injury Through Inducing HSP72 in Cerebral Microvascular Endothelial Cells. Front. Pharmacol. 2019;10:101 doi: 10.3389/fphar.2019.00101.

25. Xiao Y, Liu L, Xu A, et al. Serum fibroblast growth factor 21 levels are related to subclinical atherosclerosis in patients with type 2 diabetes. Cardiovasc. Diabetol. 2015;14:72 doi: 10.1186/s12933-015-0229-9.

26. Ferrer-Curriu G, Guitart-Mampel M, Rupérez C, et al. The protective effect of fibroblast growth factor21 in alcoholic cardiomyopathy: a role in protecting cardiac mitochondrial function. J. Pathol. 2021;253(2):198-208 doi: 10.1002/path.5573.

27. Kuroda M, Muramatsu R, Maedera N, et al. Peripherally derived FGF21 promotes remyelination in the central nervous system. J. Clin. Invest. 2017;127(9):3496-509 doi: 10.1172/jci94337.

28. Xu SL, Deng YS, Liu J, et al. Regulation of circular RNAs act as ceRNA in a hypoxic pulmonary hypertension rat model. Genomics 2021;113(1 Pt 1):11-19 doi: 10.1016/j.ygeno.2020.11.021.

29. Liu J, Deng Y, Fan Z, et al. Construction and analysis of the abnormal IncRNA-miRNA-mRNA network in hypoxic pulmonary hypertension. Biosci. Rep. 2021;41(8) doi: 10.1042/bsr20210021.

30. Liu Y, Zhang H, Li Y, et al. Long Noncoding RNA Rps4l Mediates the Proliferation of Hypoxic Pulmonary Artery Smooth Muscle Cells. Hypertension 2020;76(4):1124-33 doi: 10.1161/hypertensionaha.120.14644.

31. Xu Y, Sun Z, Wang Q, Wang T, Liu Y, Yu F. Stox1 induced the proliferation and cell cycle arrest in pulmonary artery smooth muscle cells via AKT signaling pathway. Vascul. Pharmacol. 2019;120:106568 doi: 10.1016/j.vph.2019.106568.

32. Byun S, Seok S, Kim YC, et al. Fasting-induced FGF21 signaling activates hepatic autophagy and lipid degradation via JMJD3 histone demethylase. Nature communications 2020;11(1):807 doi: 10.1038/s41467-020-14384-z.

33. Płatek T, Polus A, Góralska J, et al. Epigenetic Regulation of Processes Related to High Level of Fibroblast Growth Factor 21 in Obese Subjects. Genes 2021;12(2) doi: 10.3390/genes12020307.

34. Jeanson Y, Ribas F, Galinier A, et al. Lactate induces FGF21 expression in adipocytes through a p38MAPK pathway. Biochem. J. 2016;473(6):685-92 doi: 10.1042/bj20150808. 
35. Minard AY, Tan SX, Yang P, et al. mTORC1 Is a Major Regulatory Node in the FGF21 Signaling Network in Adipocytes. Cell reports 2016;17(1):29-36 doi: 10.1016/j.celrep.2016.08.086.

36. Zhang $Y$, Jiang $K$, Xie G, et al. FGF21 impedes peripheral myelin development by stimulating p38 MAPK/C-Jun axis. J. Cell. Physiol. 2021;236(2):1345-61 doi: 10.1002/jcp.29942.

37. Liu Z, Jiang J, Dai W, et al. MicroRNA-674-5p induced by HIF-1a targets XBP-1 in intestinal epithelial cell injury during endotoxemia. Cell death discovery 2020;6:44 doi: 10.1038/s41420-020-0280-5.

38. Li B, Zhou X, Chen L, Feng C, Li T. [Expression of microRNAs in lung homogenates in rats with chronic obstructive pulmonary disease]. Zhonghua wei zhong bing ji jiu yi xue 2014;26(12):905-9 doi: 10.3760/cma.j.issn.2095-4352.2014.12.012.

39. Oh JG, Lee P, Gordon RE, Sahoo S, Kho C, Jeong D. Analysis of extracellular vesicle miRNA profiles in heart failure. J. Cell. Mol. Med. 2020;24(13):7214-27 doi: 10.1111/jcmm.15251.

40. Lizé M, Pilarski S, Dobbelstein M. E2F1-inducible microRNA 449a/b suppresses cell proliferation and promotes apoptosis. Cell Death Differ. 2010;17(3):452-8 doi: 10.1038/cdd.2009.188.

41. Woo SY, Lee SY, Yu SL, et al. MicroRNA-7-5p's role in the 0-GIcNAcylation and cancer metabolism. Non-coding RNA research 2020;5(4):201-07 doi: 10.1016/j.ncrna.2020.11.003.

\section{Figures}



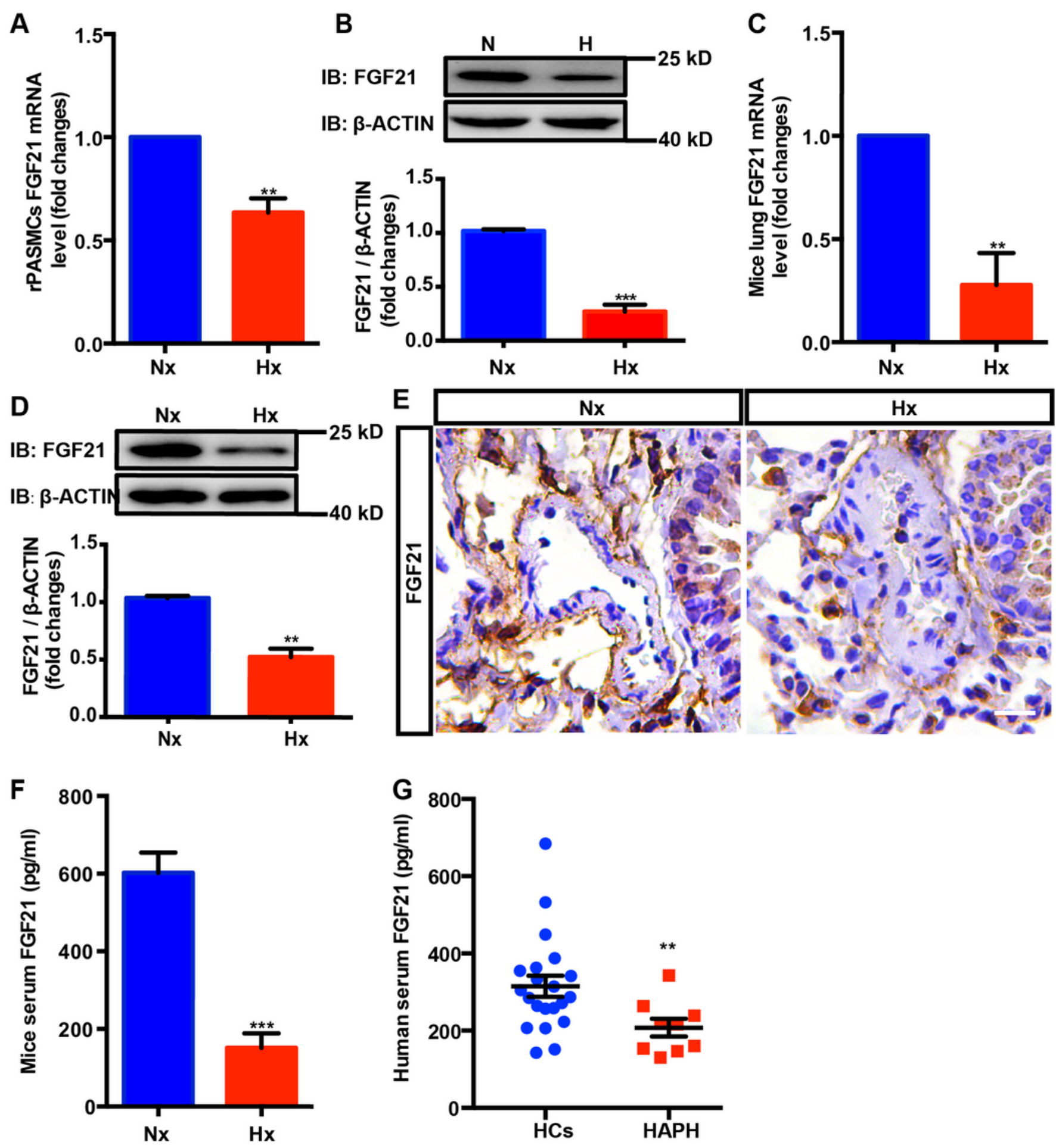

Figure 1

Expression of FGF21 is decreased in human and experimental PH. Verification of the mRNA (A) and protein (B) levels of FGF21 in rPASMCs under normoxia and hypoxia by qRT-PCR and western blotting (WB), respectively. The mRNA (C) and protein (D) levels of FGF21 in lung tissue homogenates of adult C57BL/ 6 mice under normoxia and hypoxia were verified by qRT-PCR and WB. The localization and quantification of FGF21 in lung paraffin sections of adult C57BL/6 mice under normoxia and hypoxia ( $\mathrm{n}=$ 
6, respectively) are shown by immunohistochemical images (E). ELISA was used to detect the serum FGF21 expression level of adult C57BL/ 6 mice (F) under normoxia and hypoxia or the serum FGF21 expression level of healthy individuals $(n=21)$ and HAPH patients $(n=9)(G) .{ }^{*} p<0.05,{ }^{* *} p<0.01,{ }^{* *} p<$ 0.001. Scale bars, $20 \mu \mathrm{m}$.

A

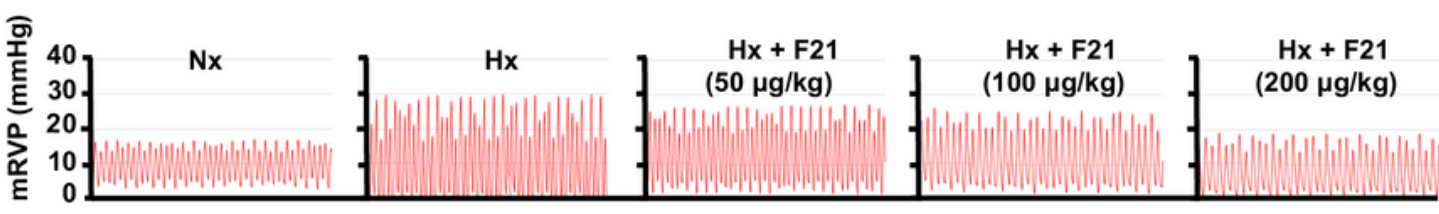

B

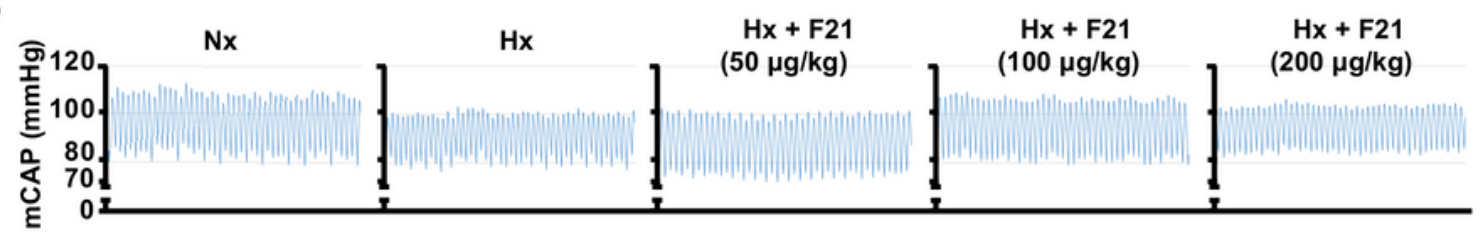

C
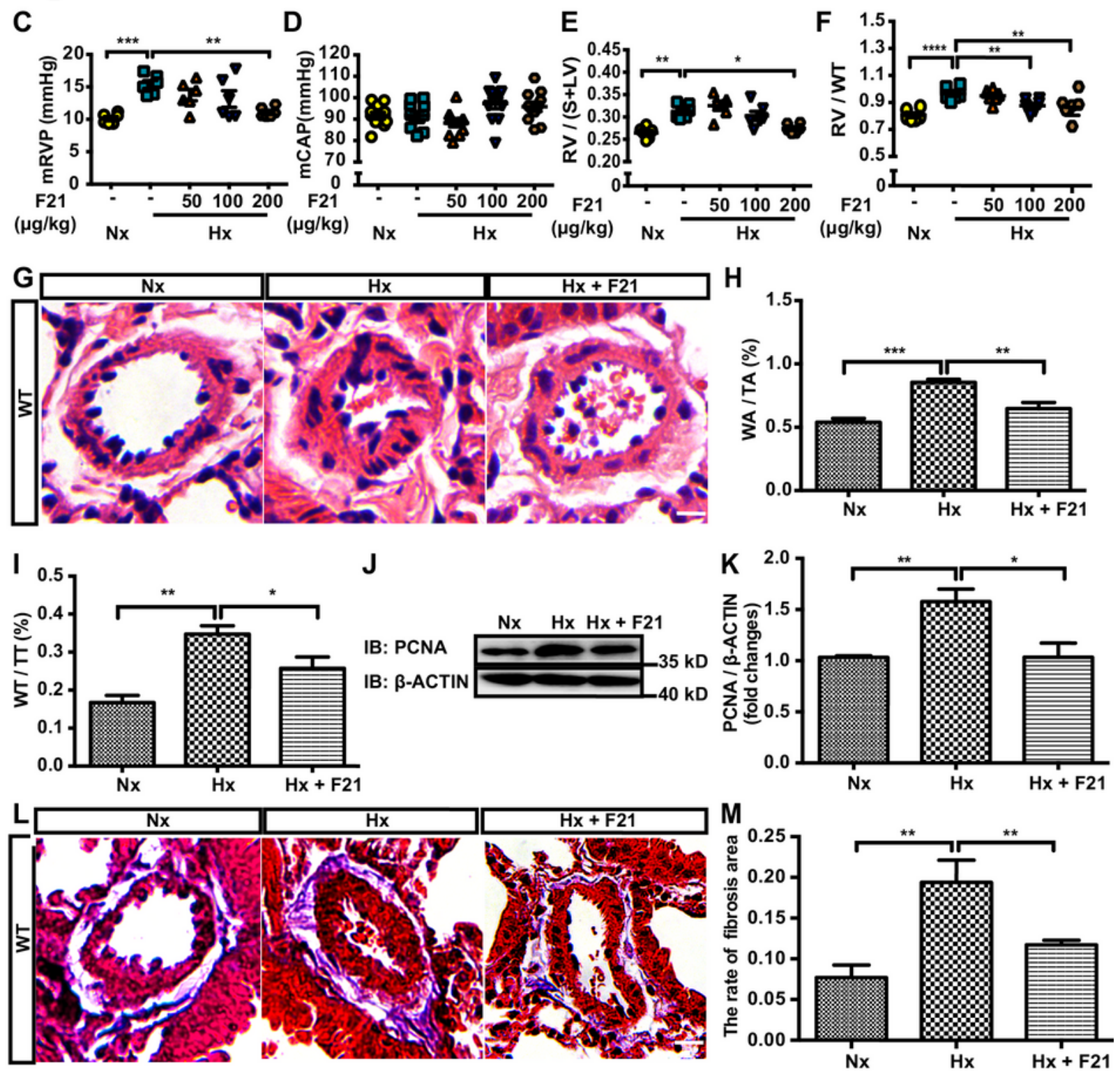

Figure 2 
PH amelioration in FGF21 treated mice. (A-D) Hemodynamic changes in control mice and HPH mice administered with different concentrations of FGF21 (0, 50, 100 and $200 \mu \mathrm{g} / \mathrm{kg})$. Summary of representative measurement results of mRVP wave (A) and mPAP (B) wave in different treatment groups. The quantitative analysis of the measurement results of RVSP and MPAP in each group is shown in (C) and $(D)(n=6)$. The right ventricular hypertrophy index is reflected by the quantitative analysis of $\mathrm{RV} /(\mathrm{S}+\mathrm{LV})(\mathrm{E})$ and RV/WT (F). (G) HE staining of pulmonary arteries. The degree of pulmonary artery remodeling in each group is reflected by WT/TT $(\mathrm{H})$ and WA/TA $(\mathrm{I}) .(\mathrm{J}-\mathrm{K})$ Western blotting to detect the protein expression level of proliferation marker PCNA in the lung tissue homogenate of each group of mice. The Masson staining $(L)$ of each group and the quantitative analysis of its fibrotic area rate $(M)$ show the degree of collagen deposition around the pulmonary arteries. ${ }^{\star} p<0.05,{ }^{*} p<0.01,{ }^{* \star} p<0.001$, $* * * p<0.0001$. Scale bars, $20 \mu \mathrm{m}$. 
A

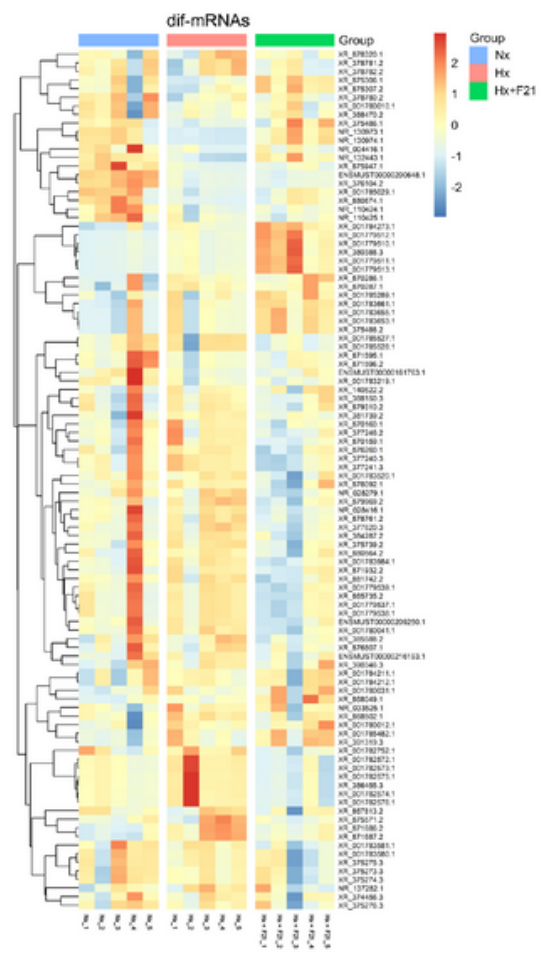

C

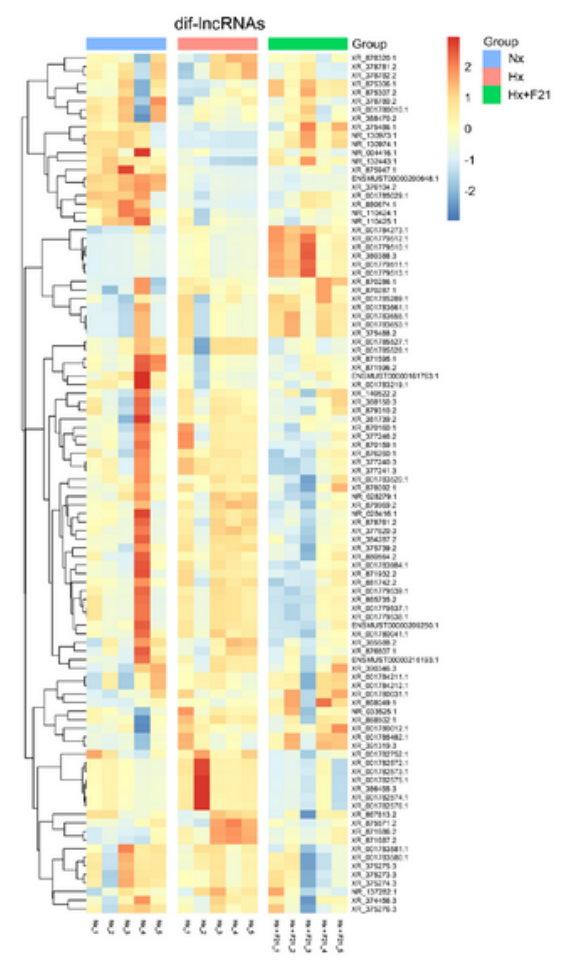

B

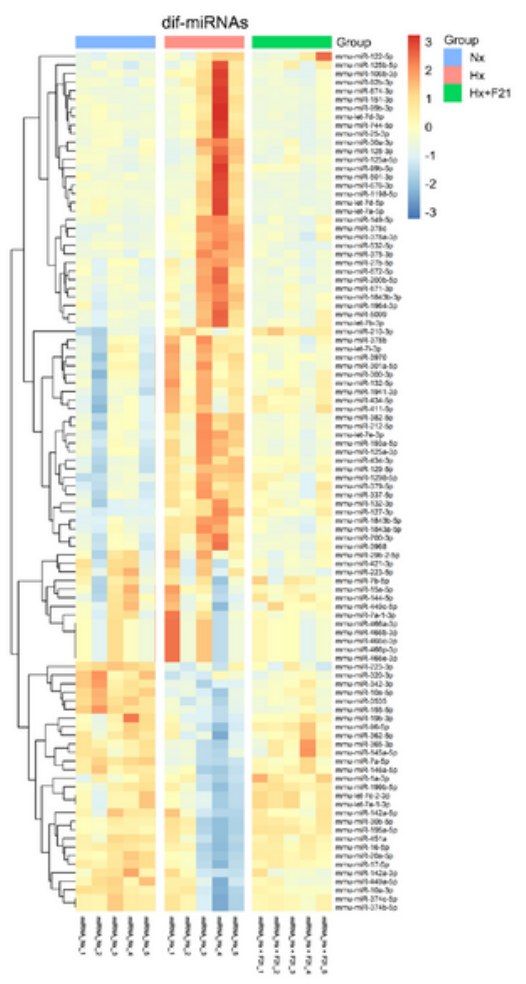

D

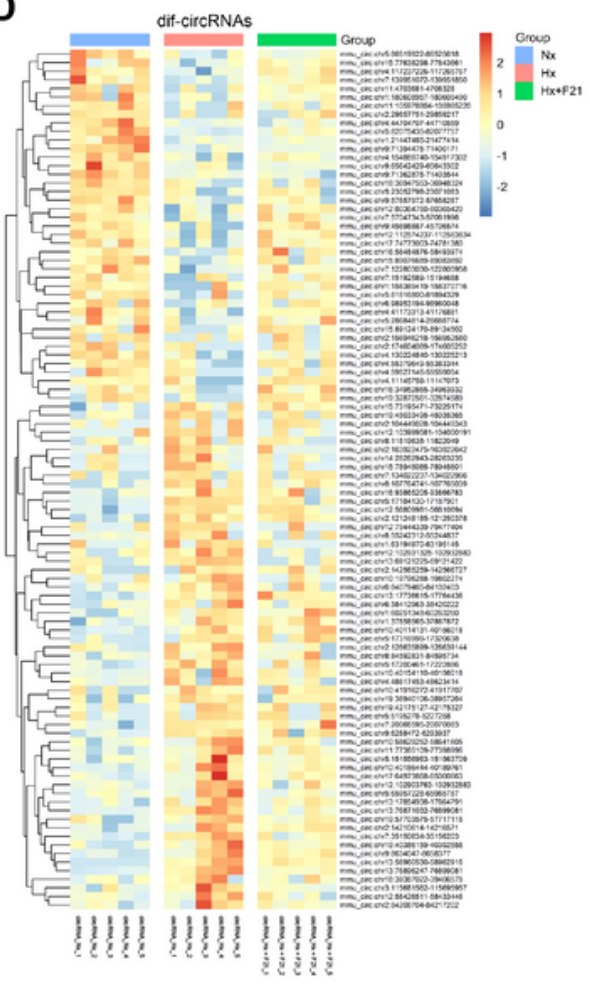

Figure 3

Heatmaps of differentially expressed RNAs.(A-D) Heatmaps of dif-mRNAs (A), dif-miRNAs (B), difIncRNAs (C), and dif-circRNAs (D). Red indicates upregulation, and blue indicates downregulation. The top 100 RNAs in $|\log 2 \mathrm{FC}|$ are displayed. 
A

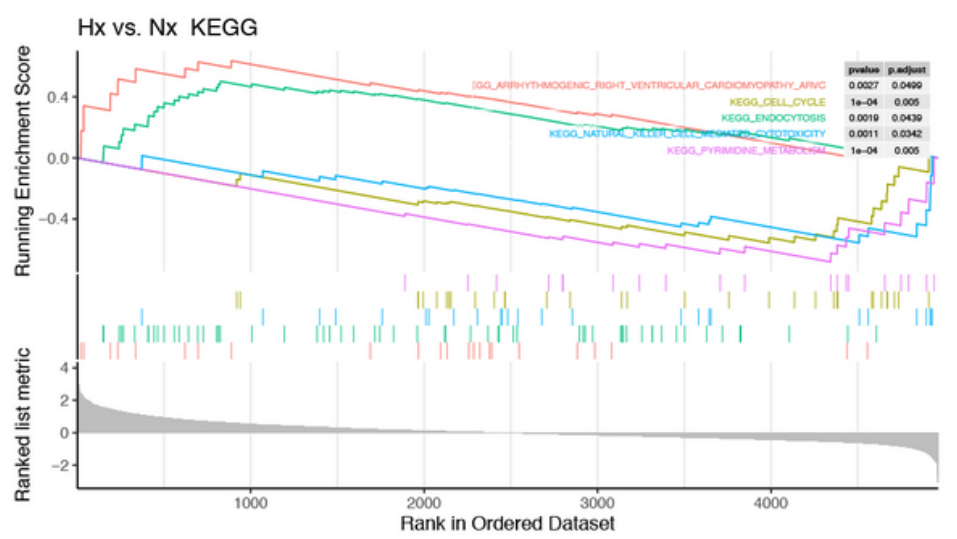

C

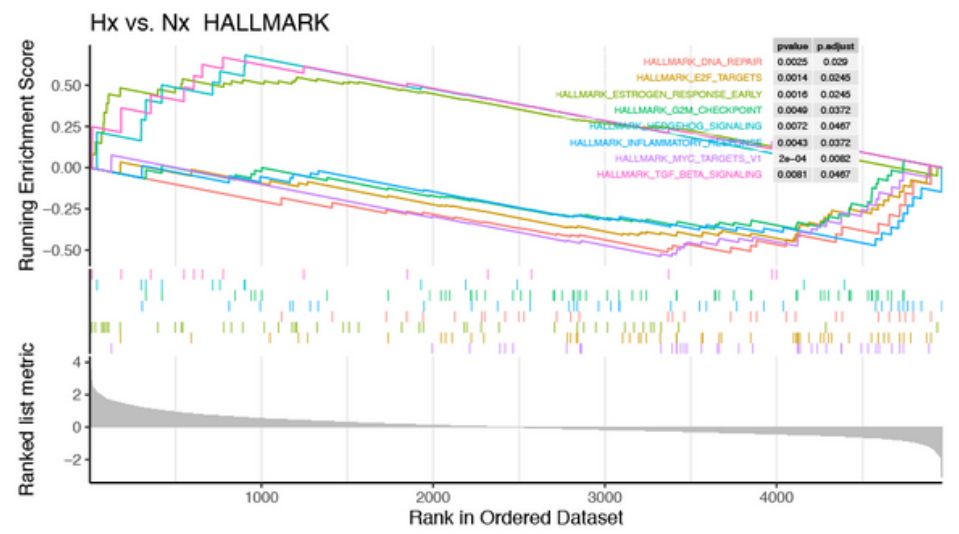

B

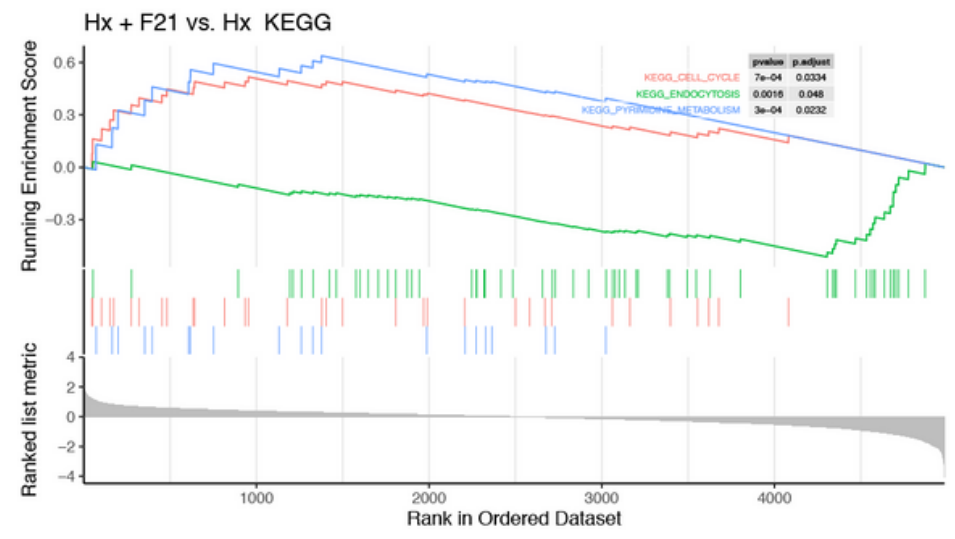

D

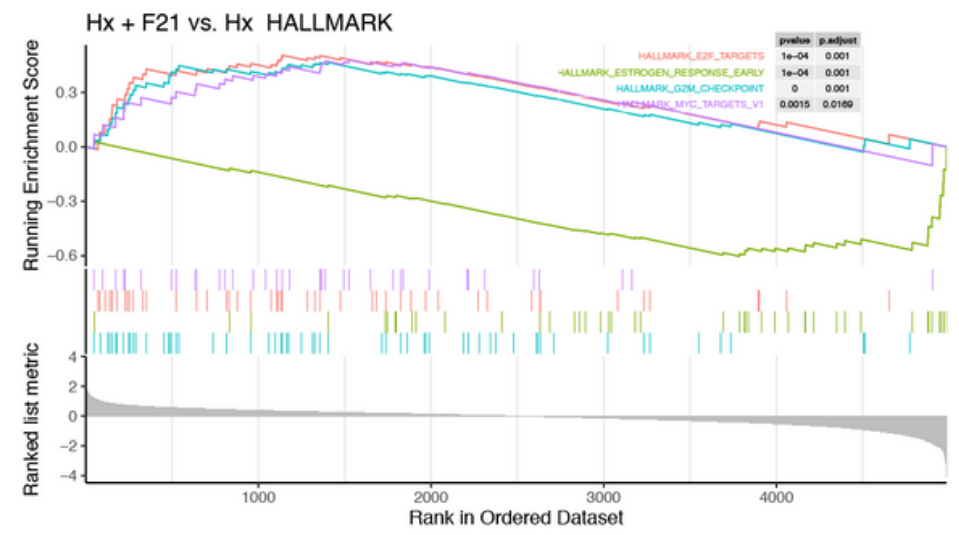

Figure 4

Gene set enrichment analysis. (A) Enriched pathways from GSEA of KEGG gene sets in Nx vs. $\mathrm{Hx},(\mathrm{B})$ Enriched pathways from GSEA of KEGG gene sets in $\mathrm{Hx} v s$. $\mathrm{Hx}+\mathrm{F} 21$. (C) The dysregulation states of pathway between different $\mathrm{Nx}$ and $\mathrm{Hx}$ are identified by GSEA hallmark gene sets, (D) The dysregulation states of pathway between different $\mathrm{Hx}$ and $\mathrm{Hx}+\mathrm{F} 21$ are identified by GSEA hallmark gene sets. 
A

down in $\mathrm{Hx}$, vs. Nx up in $\mathrm{Hx}+\mathrm{F} 21$, vs. $\mathrm{Hx}$

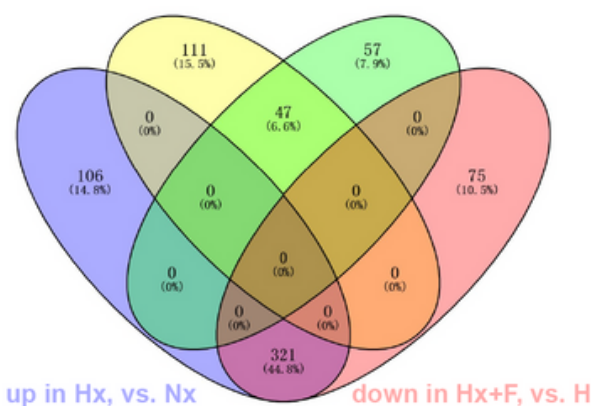

C

down in $\mathrm{Hx}$, vs. $\mathrm{Nx} \quad$ up in $\mathrm{Hx}+\mathrm{F} 21$, vs. $\mathrm{Hx}$

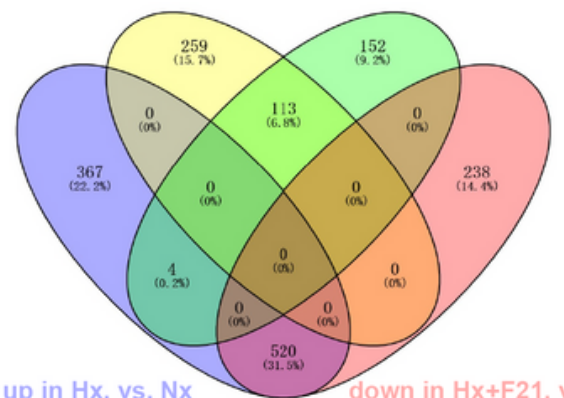

up in $\mathrm{Hx}$, vs. Nx

$\mathbf{E}$

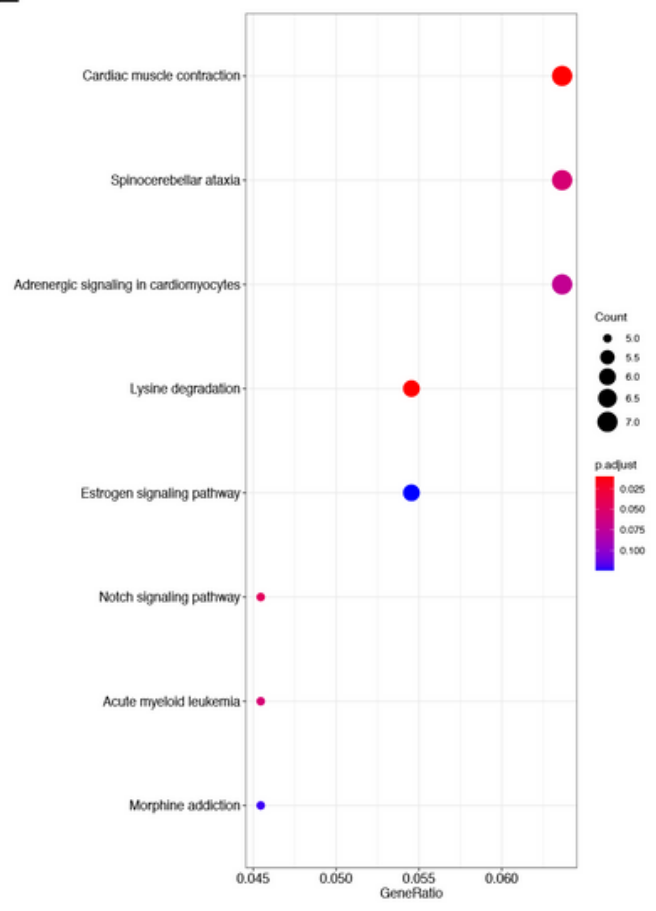

B

down in $\mathrm{Hx}$, vs. $\mathrm{Nx}$ up in $\mathrm{Hx}+\mathrm{F} 21$, vs. $\mathrm{Hx}$

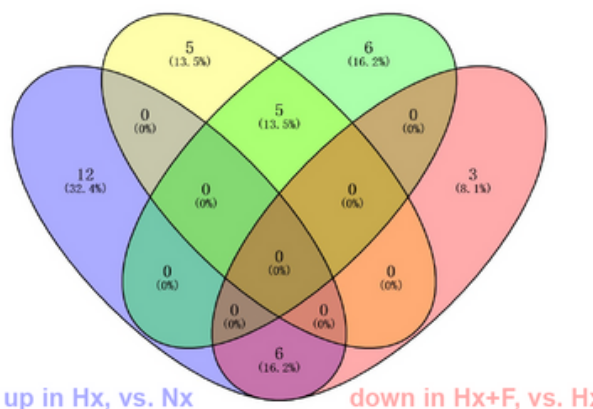

D

down in $\mathrm{Hx}$, vs. $\mathrm{Nx}$ up in $\mathrm{Hx}+\mathrm{F} 21$, vs. $\mathrm{Hx}$

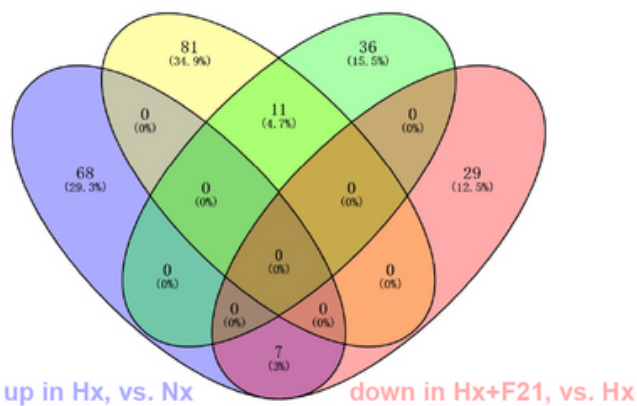

$\mathbf{F}$

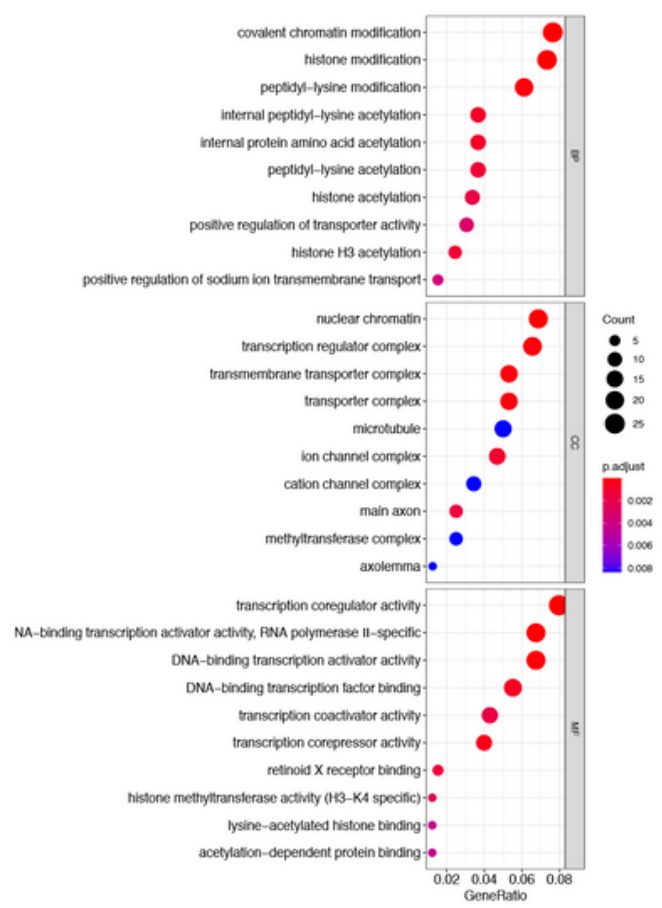

Figure 5

Screening of differentially co-expressed molecules and functional enrichment analysis. Molecules upregulated in $\mathrm{Nx} v s$. $\mathrm{Hx}$ and down-regulated in $\mathrm{Hx} v$ s. $\mathrm{Hx}+\mathrm{F} 21$, or down-regulated in $\mathrm{Nx} v s$. $\mathrm{Hx}$ and upregulated in $\mathrm{Hx} v s$. $\mathrm{Hx}+\mathrm{F} 21$ are considered to be co-expressed dif-genes (A), dif-miRNAs (B), dif-IncRNAs (C), dif-circRNAs (D). (E) KEGG pathway enrichment analysis of co-expressed dif-genes. (F) The top 10 significant GO enrichment terms. BP: biological process; CC: cell composition; MF: Molecular function. 
A

predictive targets of co-down dif-miRNAs

co-down dif-mRNAs

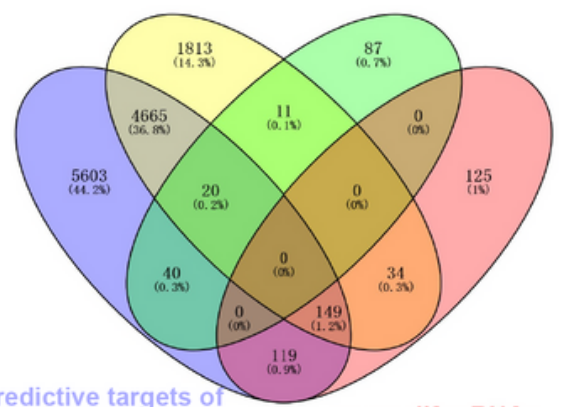

co-up dif-miRNAs

C

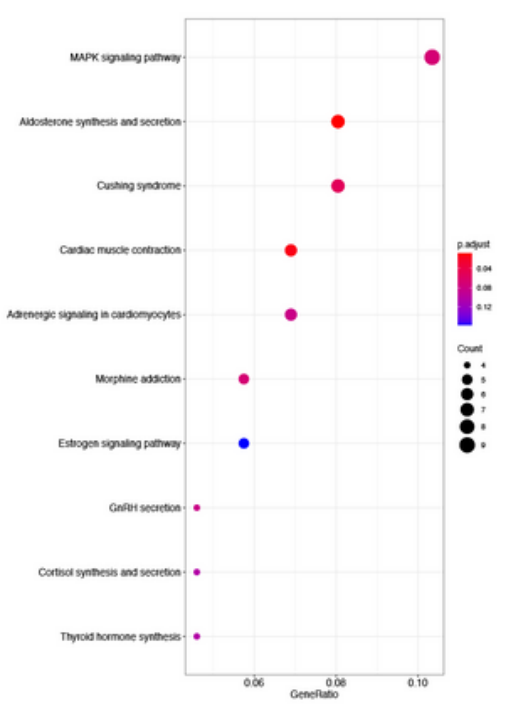

E

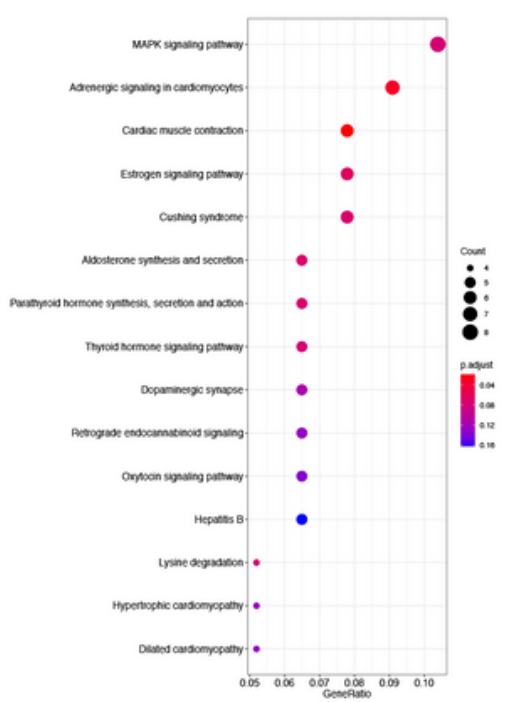

B

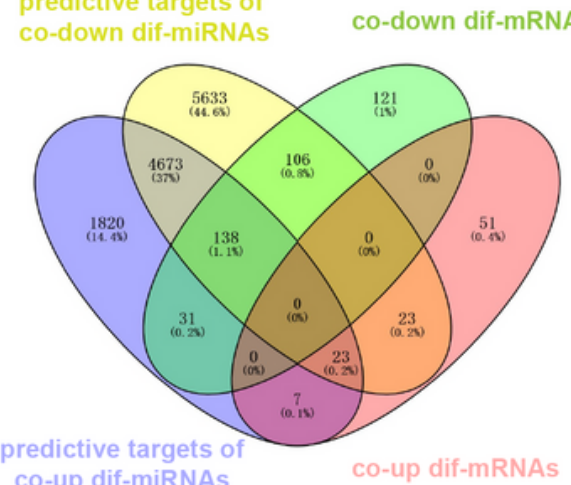

D

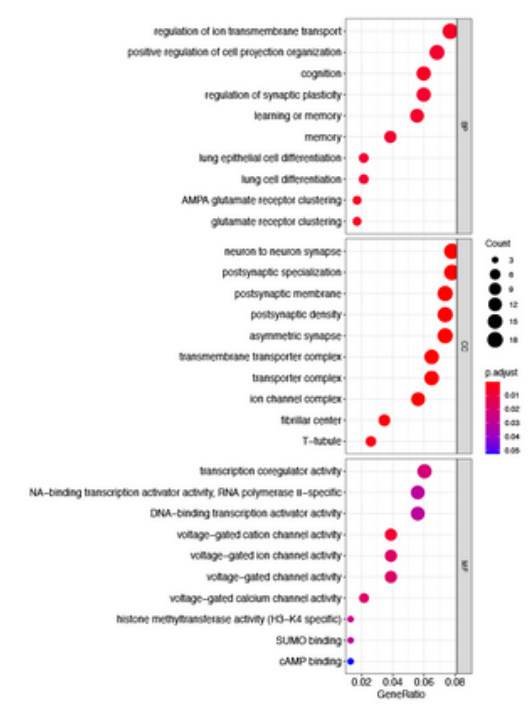

F

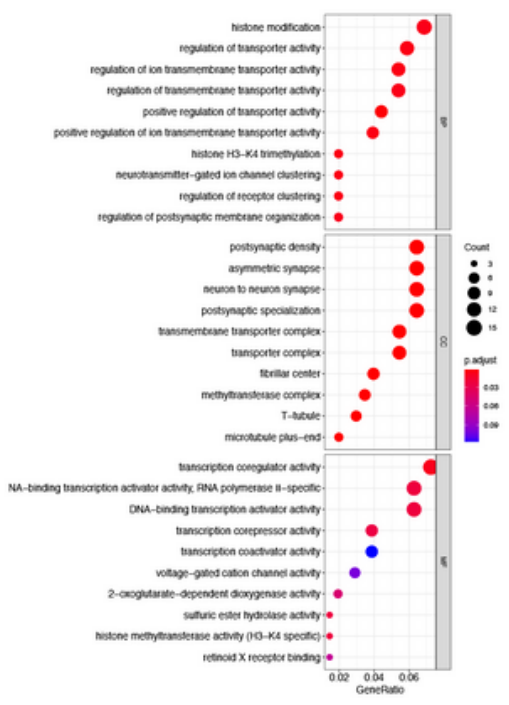

Figure 6

Functional enrichment analysis of dif-miRNA-related target genes. The Venn diagram (A) of target gene screening in $\mathrm{Nx} v s$. $\mathrm{Hx}$, and its KEGG pathway enrichment analysis (C) and the top $10 \mathrm{GO}$ (BP, MF, CC) enrichment analysis results (D). The Venn diagram (B) of target gene screening in $\mathrm{Hx} v s$. $\mathrm{Hx}+\mathrm{F} 21$, and its KEGG pathway enrichment analysis (E) and the top $10 \mathrm{GO}$ (BP, MF, CC) enrichment analysis results (F). 
A

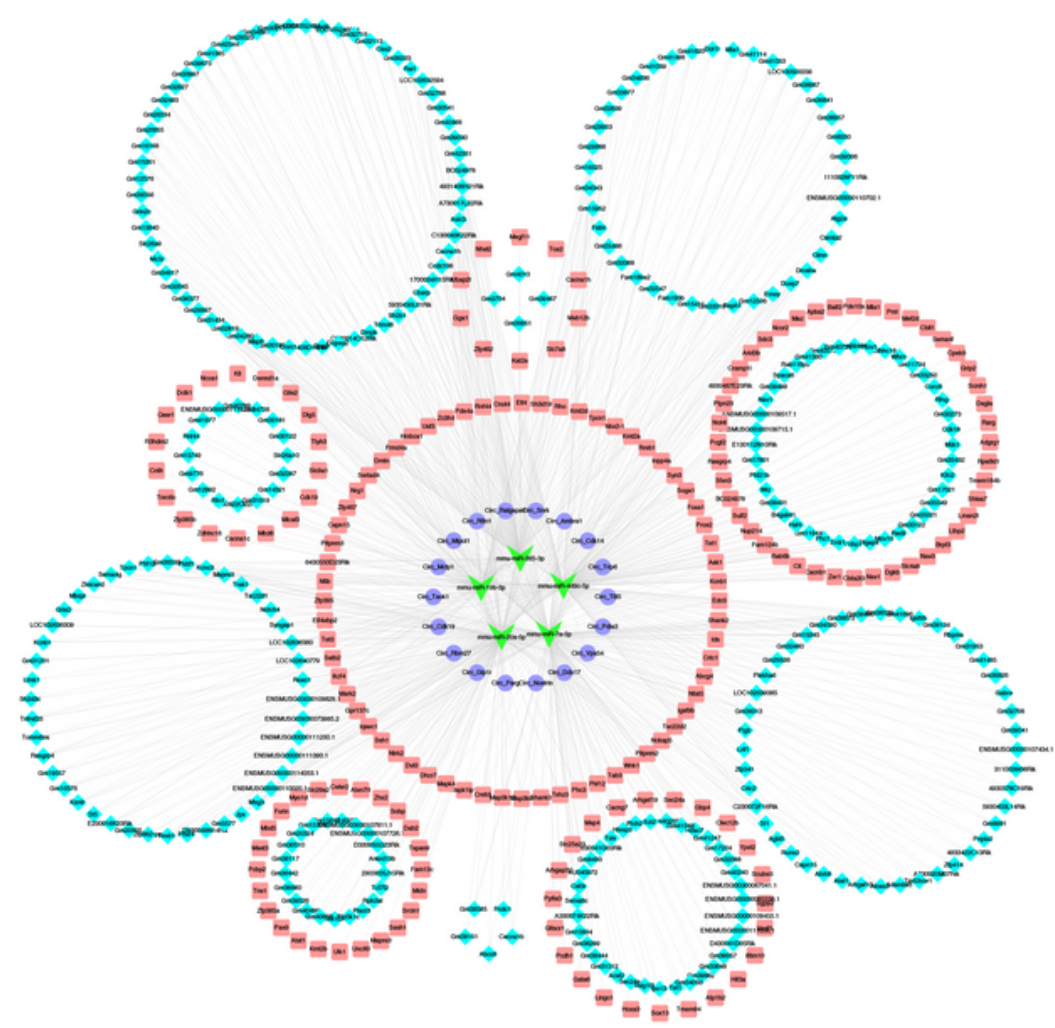

\section{B}

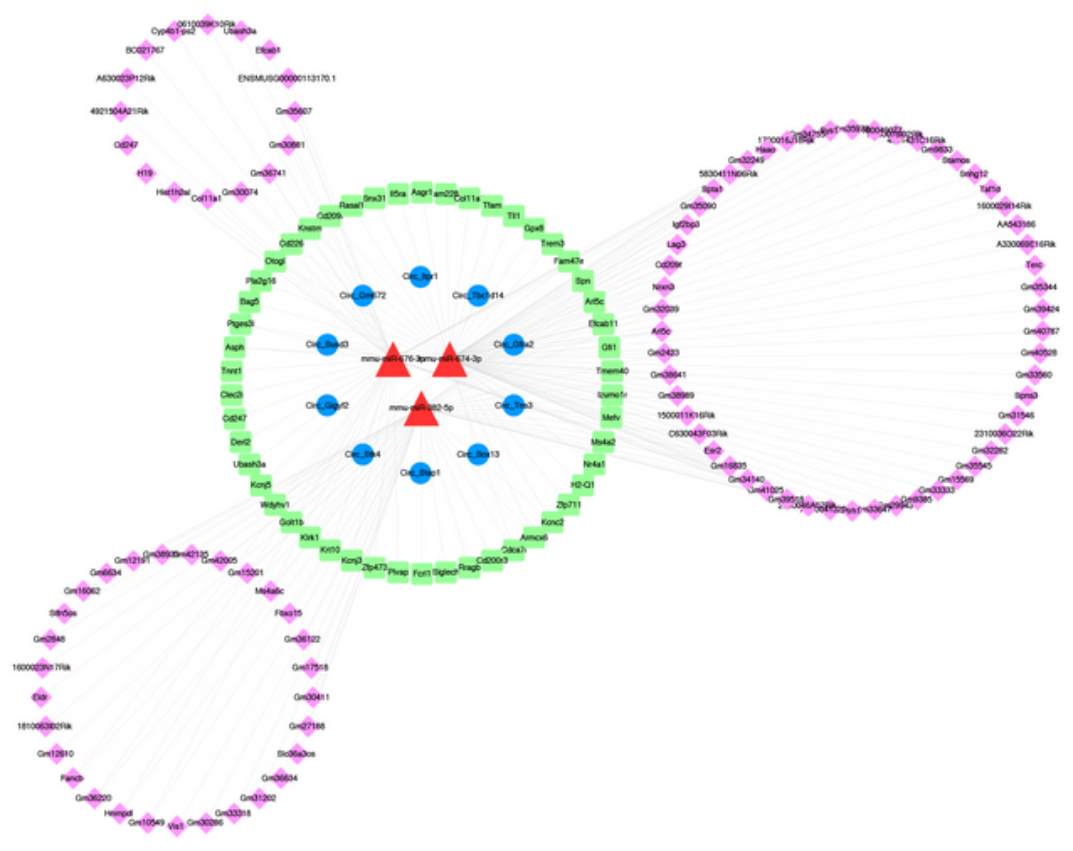

\section{Figure 7}

The competing endogenous RNA network in Nx vs. Hx. Triangles represent miRNAs, red indicates upregulation, green indicates down-regulation; squares represent genes, pink indicates up-regulation, and light green indicates down-regulation; diamonds represent IncRNAs, cyan indicates up-regulation, and lavender indicates down-regulation; rounds indicate circRNAs, purple indicates up-regulation and dark blue prompts a downward adjustment. 
A

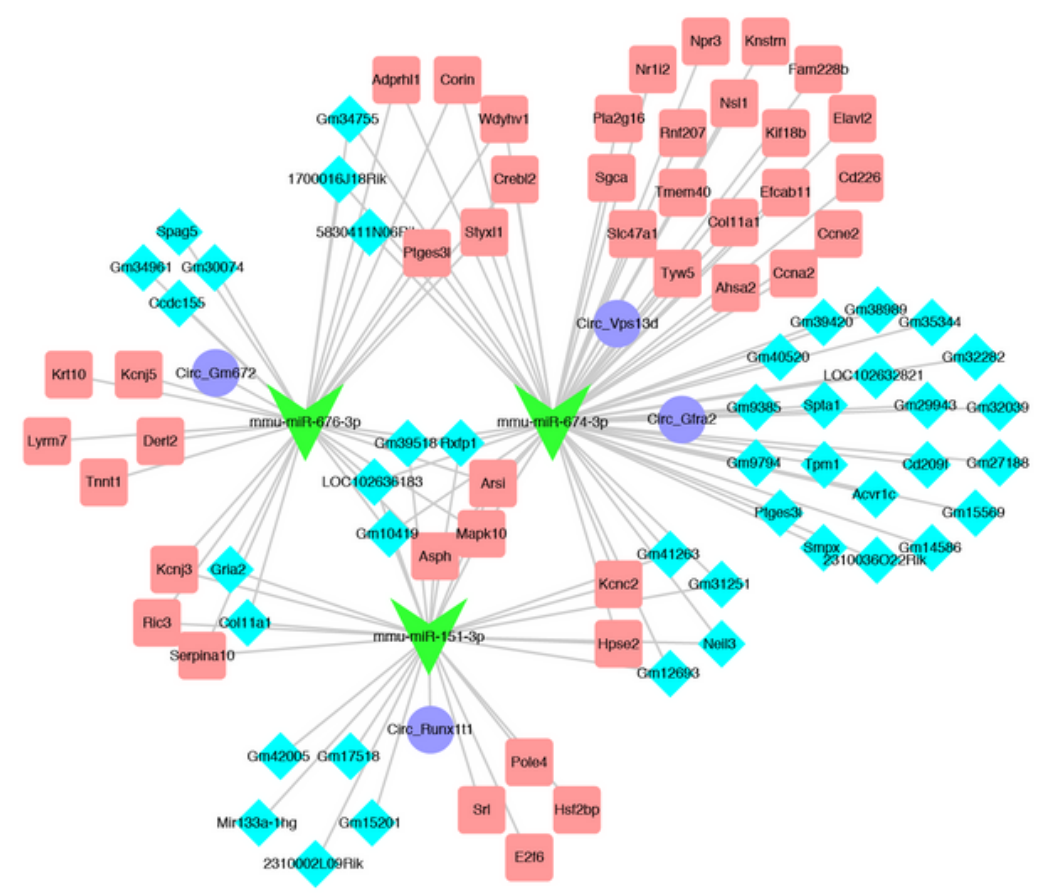

B

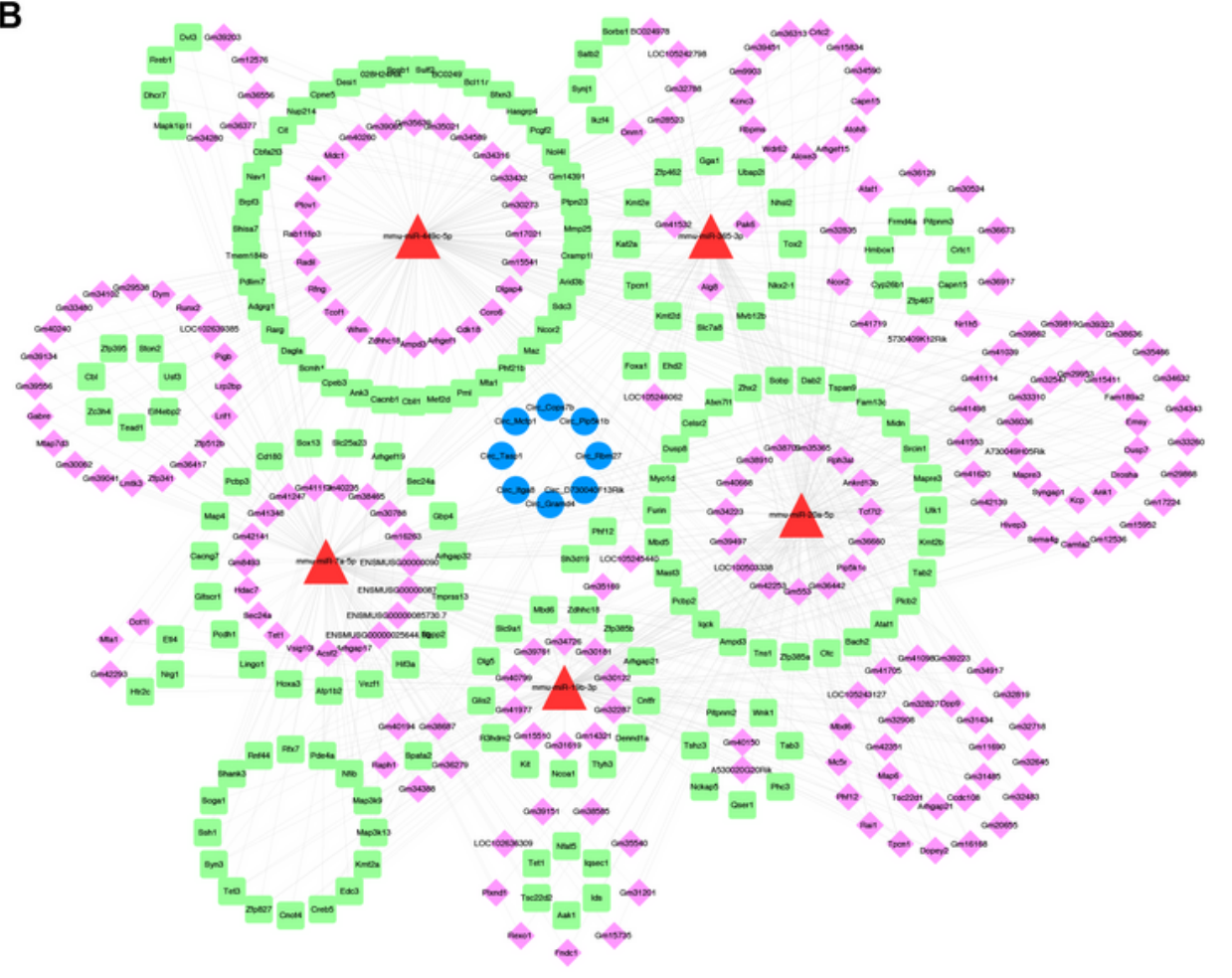

Figure 8

The competing endogenous RNA network in $\mathrm{Hx} v \boldsymbol{v} . \mathrm{Hx}+\mathrm{F} 21$. Triangles represent miRNAs, red indicates up-regulation, green indicates down-regulation; squares represent genes, pink indicates up-regulation, and light green indicates down-regulation; diamonds represent IncRNAs, cyan indicates up-regulation, and lavender indicates down-regulation; rounds indicate circRNAs, purple indicates up-regulation and dark blue prompts a downward adjustment. 

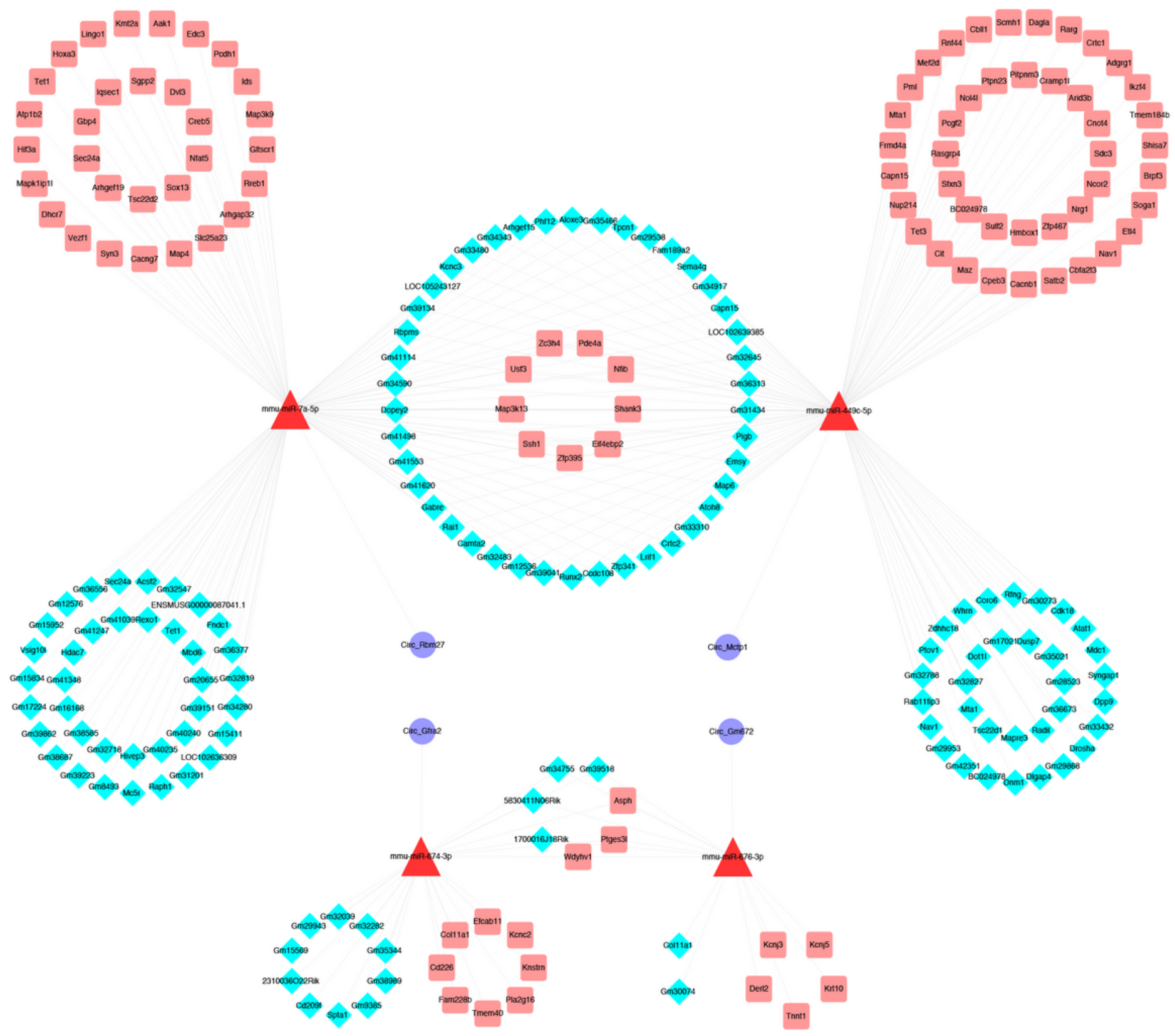

Figure 9

The competing endogenous RNA network in Nx vs. Hx vs.Hx + F21. The red triangles represent miRNAs, the pink squares represent genes, the cyan diamonds represent IncRNAs, and the purple circles represent circRNAs. 

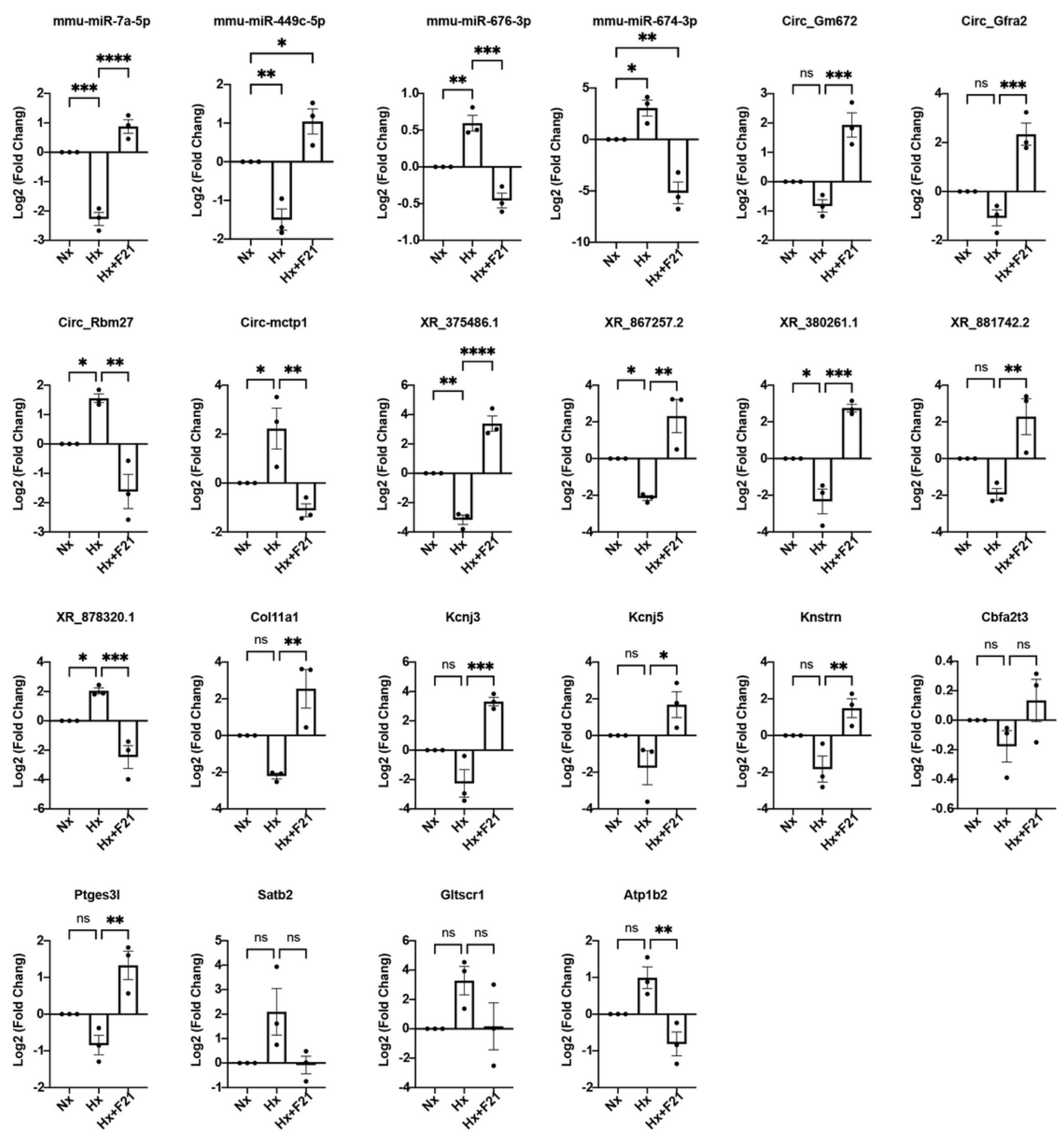

Figure 10

The expression of RNAs in Nx vs. Hx vs.Hx + F21. QRT-PCR analysis of the differentially expressed miRNAs, IncRNAs, circRNAs, mRNAs in Nx vs. Hx vs.Hx + F21. ${ }^{\star} p<0.05,{ }^{* *} p<0.01,{ }^{* *} p<0.001,{ }^{* * * *} p<$ 0.0001 . 


\section{Supplementary Files}

This is a list of supplementary files associated with this preprint. Click to download.

- Additionalfile1.tif

- Additionalfile2.tif

- Additionalfile3.tif

- DATAS1.xIsx

- DATAS10.xlsx

- DATAS11.xlsx

- DATAS12.xlsx

- DATAS13.xlsx

- DATAS14.xlsx

- DATAS15.xlsx

- DATAS16.xlsx

- DATAS17.xlsx

- DATAS18.xlsx

- DATAS2.xIsx

- DATAS3.xIsx

- DATAS4.xIsx

- DATAS5.xIsx

- DATAS6.xIsx

- DATAS7.xIsx

- DATAS8.xIsx

- DATAS9.xIsx 